TRANSACTIONS OF THE

AMERICAN MATHEMATICAL SOCIETY

Volume 357, Number 5, Pages 2081-2117

S 0002-9947(04)03604-9

Article electronically published on December 28, 2004

\title{
GENERALIZED SPHERICAL FUNCTIONS ON REDUCTIVE $p$-ADIC GROUPS
}

\author{
JING-SONG HUANG AND MARKO TADIĆ
}

\begin{abstract}
Let $G$ be the group of rational points of a connected reductive $p$-adic group and let $K$ be a maximal compact subgroup satisfying conditions of Theorem 5 from Harish-Chandra (1970). Generalized spherical functions on $G$ are eigenfunctions for the action of the Bernstein center, which satisfy a transformation property for the action of $K$. In this paper we show that spaces of generalized spherical functions are finite dimensional. We compute dimensions of spaces of generalized spherical functions on a Zariski open dense set of infinitesimal characters. As a consequence, we get that on that Zariski open dense set of infinitesimal characters, the dimension of the space of generalized spherical functions is constant on each connected component of infinitesimal characters. We also obtain the formula for the generalized spherical functions by integrals of Eisenstein type. On the Zariski open dense set of infinitesimal characters that we have mentioned above, these integrals then give the formula for all the generalized spherical functions. At the end, let as mention that among others we prove that there exists a Zariski open dense subset of infinitesimal characters such that the category of smooth representations of $G$ with fixed infinitesimal character belonging to this subset is semi-simple.
\end{abstract}

\section{INTRODUCTION}

In the case of a real reductive group $G$ with complexified Lie algebra $\mathfrak{g}$, zonal spherical functions are defined as eigenfunctions for the action of the center $Z(\mathfrak{g})$ of the universal enveloping algebra $U(\mathfrak{g})$ of $\mathfrak{g}$ on the smooth functions on $G$ which are constant on double $K$-classes, where $K$ denotes a maximal compact subgroup of $G$. In this way, one deals with differential equations and eigenfunctions of differential operators. Generalized spherical functions on real reductive groups are a natural generalization of zonal spherical functions (see [HOW]).

In the representation theory of reductive $p$-adic groups the Bernstein center ([BD]) plays the role of the center of the universal enveloping algebra in the representation theory of real reductive groups. The Bernstein center shows up in a number of important problems, but its role in representation theory is far from being well understood.

Received by the editors March 31, 2003 and, in revised form, January 2, 2004.

2000 Mathematics Subject Classification. Primary 22E50, 22E35.

Key words and phrases. Reductive $p$-adic group, generalized spherical function, Bernstein center, infinitesimal character.

The first author was partially supported by Hong Kong Research Grant Council Competitive Earmarked Research Grant. The second author was partly supported by Croatian Ministry of Science and Technology grant \# 37108. 
The construction of the Bernstein center of a reductive $p$-adic group ([BD]$)$ opened the possibility of also studying generalized spherical functions in the $p$-adic case. In this paper we define and study basic properties of generalized spherical functions in the case of a reductive $p$-adic group (they are defined as eigenfunctions of the Bernstein center, which satisfy a transformation property for the action of a maximal compact subgroup). This paper may be viewed as a contribution to the further understanding of the Bernstein center.

Let $G$ be the group of rational points of a connected reductive group defined over a $p$-adic field $F$. Fix a minimal parabolic subgroup $P_{\emptyset}$ defined over $F$ and let $K$ be a maximal compact subgroup of $G$ satisfying $G=P_{\emptyset} K$. Let $\left(\tau_{1}, V_{\tau_{1}}\right),\left(\tau_{2}, V_{\tau_{2}}\right)$ be finite-dimensional representations of $K$. Denote

$$
V=\operatorname{Hom}_{\mathbb{C}}\left(V_{\tau_{2}}, V_{\tau_{1}}\right)
$$

and

$$
\tau=\left(\tau_{1}, \tau_{2}\right) .
$$

Then generalized spherical functions of type $\tau$ (or $\tau$-spherical eigenfunctions) are mappings $f: G \rightarrow V$ which satisfy $f\left(k_{1} x k_{2}\right)=\tau_{1}\left(k_{1}\right) f(x) \tau_{2}\left(k_{2}\right)$ for all $k_{1}, k_{2} \in K$, $g \in G$, and which are eigenfunctions for the action of the Bernstein center. If $\omega$ is the corresponding infinitesimal character of the Bernstein center of $G$, then the space of all generalized spherical functions of type $\tau$ corresponding to the infinitesimal character $\omega$ will be denoted by

$$
\mathcal{E}_{\omega}(G, \tau) .
$$

In this paper we first show that these spaces are finite dimensional (Corollary 5.5). We show how one can get all the generalized spherical functions from a single admissible representation with contragredient infinitesimal character $\tilde{\omega}$ (Propositions 5.4 and 6.1). After this, we show that on a Zariski open dense set of infinitesimal characters, one can choose the above representation to be irreducible (in this case there exists exactly one such representation and it has a simple description). We also compute dimensions of spaces of generalized spherical functions for infinitesimal characters in this Zariski open dense set (Theorem 6.4). If $\omega$ is an infinitesimal character of a parabolically induced representation $\operatorname{Ind}_{P}^{G}(\rho)$, where $\rho$ is an irreducible cuspidal representation of Levi factor $M$ of $P$, then the dimension of the generalized spherical functions for $\omega$ in a Zariski open dense set of infinitesimal characters is given by

$$
\operatorname{dim}_{\mathbb{C}}\left(\mathcal{E}_{\omega}(G, \tau)\right)=\operatorname{dim}_{\mathbb{C}}\left(\operatorname{Hom}_{M \cap K}\left(\tau_{1}^{N \cap K}, \tilde{\rho}\right)\right) \operatorname{dim}_{\mathbb{C}}\left(\operatorname{Hom}_{M \cap K}\left(\tau_{2}^{N \cap K}, \tilde{\rho}\right)\right),
$$

where $\tilde{\rho}$ denotes the contragredient representation of $\rho, N$ denotes the unipotent radical of $P$ and $\tau_{i}^{N \cap K}$ denote the $N \cap K$-invariants in $\tau_{i}$ for $i=1,2$. On this Zariski open dense set of infinitesimal characters, the dimension of the space of generalized functions is constant on each connected component of infinitesimal characters.

We also obtain the formula for the generalized spherical functions in terms of integrals of Eisenstein type. On the Zariski open dense set of infinitesimal characters that we mentioned above, these integrals then give the formula for all the generalized spherical functions. Further, we prove that for a Zariski open dense subset of infinitesimal characters, the category of smooth representations with fixed infinitesimal character belonging to this subset is semi-simple.

This paper is the $p$-adic analogue of a part of the basic results for real reductive groups obtained in [HOW]. It is in tune with what Harish-Chandra called the Lefschetz principal, which says that whatever is true for real reductive groups is 
also true for $p$-adic groups. There are many not so surprising similarities between our proofs for the $p$-adic case and that for the real case in [HOW] (but there are a number of differences). The Hecke algebra $\mathcal{H}(G)$ of compactly supported smooth functions on a $p$-adic group often plays a role similar to that of the universal enveloping algebra $U(\mathfrak{g})$ of a real group. The Bernstein center $\mathfrak{Z}(G) \cong \operatorname{End}_{G \times G}(\mathcal{H}(G))$ plays the same role as the center $Z(\mathfrak{g})$ of $U(\mathfrak{g})$ in the case of real groups. The main tools that we use here are the techniques of the Bernstein center ([B]] and [BDK] $)$.

At the end of this Introduction we shall give a brief account of the paper. In the second section we introduce notations regarding actions of the group $G$ on spaces of functions on $G$. The third section recalls the definition of the Bernstein center. We prove also here some simple facts about actions of the Bernstein center needed in the sequel. Generalized spherical functions are defined in the fourth section. The fifth section starts with the study of spherical functions and their dimensions. In this section we prove that spaces of generalized spherical functions are finite dimensional. In the sixth section we prove the formula for dimension of spaces of generalized spherical functions for infinitesimal characters in a Zariski open dense set of infinitesimal characters. The formula for the generalized spherical functions by integrals of Eisenstein type is obtained in the seventh section. In the last section we prove that for a Zariski open dense subset of infinitesimal characters, the category of smooth representations with fixed infinitesimal character belonging to this subset is semi-simple.

\section{Actions on FUnCtions}

In this section we shall denote by $G$ a locally compact totally disconnected group. Let $V$ be an $n$-dimensional complex vector space. The dual space of $V$ will be denoted by $V^{\prime}$. We shall fix a basis $e_{1}, e_{2}, \ldots, e_{n}$ of $V$. Let $e_{1}^{\prime}, e_{2}^{\prime}, \ldots, e_{n}^{\prime}$ denote the dual basis in $V^{\prime}$.

The space of all continuous (resp. locally constant) functions from $G$ into $V$ will be denoted by $C(G, V)\left(\operatorname{resp} C^{\infty}(G, V)\right)$. The space $C(G, \mathbb{C})$ will be simply denoted by $C(G)$. We shall consider left and right actions $L$ and $R$ of $G$ on $C(G, V)$ (here $\left(L_{x} \varphi\right)(g)=\varphi\left(x^{-1} g\right)$ and $\left.\left(R_{x} \varphi\right)(g)=\varphi(g x)\right)$. The smooth part of this $G \times$ $G$-representation will be denoted by $C(G, V)^{(s, s)}$. This space consists of all the functions $\varphi: G \rightarrow V$ for which there exists an open compact subgroup $H$ of $G$ such that $\varphi$ is constant on each double $H$-class.

For a linear operator $A: W_{1} \rightarrow W_{2}$ between (complex) vector spaces, we shall denote by $\Lambda_{A}: C\left(G, W_{1}\right) \rightarrow C\left(G, W_{2}\right)$ the linear operator defined by $\left(\Lambda_{A}(\varphi)\right)(g)=$ $A(\varphi(g))$ (i.e. $\left.\Lambda_{A}(\varphi)=A \circ \varphi\right)$. Then $\Lambda_{A}$ intertwines the left actions $L$ on $C\left(G, W_{1}\right)$ and $C\left(G, W_{2}\right)$, as well as the right action $R$ (for example, $\left(L_{x}\left(\Lambda_{A}(\varphi)\right)\right)(g)=$ $\left.\left(\Lambda_{A}(\varphi)\right)\left(x^{-1} g\right)=A\left(\varphi\left(x^{-1} g\right)\right)=A\left(\left(L_{x} \varphi\right)(g)\right)=\left(\Lambda_{A}\left(L_{x} \varphi\right)\right)(g)\right)$.

The following two simple particular cases of linear operators will be important for us. Let $v^{\prime} \in V^{\prime}$. Then

$$
\Lambda_{v^{\prime}}: C(G, V) \rightarrow C(G), \quad \varphi \mapsto v^{\prime} \circ \varphi
$$

Further, for $v \in V$ consider the mapping $v^{\#}: \mathbb{C} \rightarrow V, c \mapsto c v$. Then

$$
\Lambda_{v \#}: C(G) \rightarrow C(G, V), \quad f \mapsto(g \mapsto f(g) v) .
$$


The bilinear mapping $(f, v) \mapsto(g \mapsto f(g) v), \quad C(G) \times V \rightarrow C(G, V)$ induces a natural isomorphism

$$
I_{\otimes}: C(G) \otimes V \rightarrow C(G, V), \quad I_{\otimes}\left(\sum f_{i} \otimes v_{i}\right)(g)=\sum f_{i}(g) v_{i},
$$

i.e.

$$
I_{\otimes}\left(\sum f_{i} \otimes v_{i}\right)=\sum \Lambda_{v_{i}^{\#}}\left(f_{i}\right)
$$

Obviously,

$$
\varphi=I_{\otimes}\left(\sum_{i=1}^{n} e_{i}^{\prime} \circ \varphi \otimes e_{i}\right)=I_{\otimes}\left(\sum_{i=1}^{n} \Lambda_{e_{i}^{\prime}}(\varphi) \otimes e_{i}\right)=\sum_{i=1}^{n} \Lambda_{e_{i}^{\#}}\left(\Lambda_{e_{i}^{\prime}}(\varphi)\right) .
$$

We shall consider the trivial action of $G$ on $V$. Then $G$ acts on $C(G) \otimes V$ in two natural ways, one coming from the left and the other from the right action on $C(G)$. Then $I_{\otimes}$ is an intertwining for both actions. Using $I_{\otimes}$, we shall identify $C(G) \otimes V$ with $C(G, V)$.

For a smooth representation $(\pi, X)$ of $G$ the contragredient representation is denoted by $(\tilde{\pi}, \tilde{X})$.

Let us now recall claim (2) from Lemma I.6.1 of $[\mathrm{W}]$ :

2.1. Lemma. For $f \in C(G)^{(s, s)}$ the following conditions are equivalent:

(1) The subrepresentation generated by $f$ with respect to the right action of $G$ is admissible.

(2) The subrepresentation generated by $f$ with respect to the left action of $G$ is admissible.

This is equivalent to the fact that there exists an admissible representation $(\pi, X)$ of $G, x_{i} \in X$ and $\tilde{x}_{i} \in \tilde{X}, i=1, \ldots, k$, such that $f(g)=\sum_{i=1}^{k} \tilde{x}_{i}\left(\pi(g) x_{i}\right)$ for $g \in G$ (i.e. $f$ is a sum of matrix coefficients of an admissible representation).

The subspace generated by all the matrix coefficients

$$
g \mapsto\left\langle\pi\left(g^{-1}\right) v, \tilde{v}\right\rangle
$$

of all admissible representations $(\pi, X)$ of $G$ is denoted by $\mathcal{A}(G)$. Note that (1) and (2) characterize $\mathcal{A}(G)$ in different ways.

Denote by $\mathcal{A}(G, V)$ the space of all $\varphi \in C(G, V)^{(s, s)}$ such that $\varphi$ generates an admissible representation for the left action. Obviously, by Lemma $2.1 \mathcal{A}(G) \otimes V \subseteq$ $\mathcal{A}(G, V)$ since $\Lambda_{v \#}$ is an intertwining. From the other side, by Lemma 2.1 we know that $\Lambda_{v^{\prime}}(\varphi) \in \mathcal{A}(G)$ if $\varphi \in \mathcal{A}(G, V)$, since $\Lambda_{v^{\prime}}$ intertwines (left) representations. Thus

$$
\mathcal{A}(G) \otimes V=\mathcal{A}(G, V) .
$$

In the same way we could get that $\mathcal{A}(G, V)$ is the set of all $\varphi \in C(G, V)^{(s, s)}$ such that $\varphi$ generates an admissible representation for the right action. Obviously, $\mathcal{A}(G) \otimes V$ and $\mathcal{A}(G, V)$ are invariant for both actions of $G$ (and isomorphic as $G \times G$-representations).

For an admissible representation $(\pi, X)$ of $G$ we shall denote by

$$
\mathcal{A}(\pi)
$$

the vector subspace spanned by all matrix coefficients of $\pi$. Then $\mathcal{A}(\pi)$ is invariant for the left and the right action of $G$. 
Let $(\pi, X)$ be a smooth representation of $G$ and let $Y$ be a complex vector space. Then we consider an action $L$ on $\operatorname{Hom}_{\mathbb{C}}(X, Y)$ defined by

$$
(L(g) A)(x)=A\left(\pi\left(g^{-1}\right) x\right), \quad g \in G, A \in \operatorname{Hom}_{\mathbb{C}}(X, Y), x \in X .
$$

The smooth part of this representation will be denoted by $\operatorname{Hom}_{\mathbb{C}}(X, Y)^{(s)}$. Note that $\left(L, \operatorname{Hom}_{\mathbb{C}}(X, \mathbb{C})^{(s)}\right)=(\tilde{\pi}, \tilde{X})$.

Sometimes we shall consider the action $\mathcal{L}$ of $G$ on $\operatorname{Hom}_{\mathbb{C}}(Y, X)$ given by

$$
\left(\mathcal{L}_{g} A\right)(y)=\pi(g) A(y), \quad g \in G, A \in \operatorname{Hom}_{\mathbb{C}}(Y, X), y \in Y .
$$

Note that $\operatorname{Hom}_{\mathbb{C}}(Y, X)$ is a smooth representation of $G$ if $Y$ is finite dimensional.

\section{The Bernstein center $\mathfrak{Z}(G)$ and infinitesimal characters}

In the rest of the paper we shall denote by $G$ the group of rational points of a connected reductive group over a local field $F$. The modulus character of $F$ will be denoted by $\mid{ }_{F}$. Let $G^{0}$ be the group of all $g \in G$ such that $|\chi(g)|_{F}=1$ for all rational characters $\chi$ of $G$. Then

$$
G / G^{0}
$$

is a free $\mathbb{Z}$-module of finite rank (the rank is equal to the dimension of the maximal split torus in the center of $G$, i.e. to the split rank of $G$ ).

A character $\chi: G \rightarrow \mathbb{C}^{\times}$is called unramified if it is trivial on $G^{0}$. Obviously, one can identify the group of all unramified characters of $G$ with the group

$$
\Psi(G)=\operatorname{Hom}_{\mathbb{Z}}\left(G / G^{0}, \mathbb{C}^{\times}\right) .
$$

Choose any basis $b_{1}, b_{2}, \ldots, b_{l}$ of the free $\mathbb{Z}$-module $G / G^{0}$ (here $l$ is the split rank of $G)$. Then $\chi \mapsto\left(\chi\left(b_{1}\right), \chi\left(b_{2}\right), \ldots, \chi\left(b_{l}\right)\right)$ defines a group isomorphism of $\Psi(G)$ onto $\left(\mathbb{C}^{\times}\right)^{l}$. We supply $\Psi(G)$ with the (unique) structure of a complex algebraic variety, such that the above group isomorphism is also an isomorphism of algebraic varieties. In this way $\Psi(G)$ becomes a commutative complex algebraic group. Obviously, this structure does not depend on the choice of the basis $b_{1}, b_{2}, \ldots, b_{l}$.

Let $\tilde{G}$ be the set of equivalence classes of all the irreducible smooth representations of $G$. Fix $\pi \in \tilde{G}$. Consider

$$
\chi \mapsto \chi \pi, \quad \Psi(G) \rightarrow \tilde{G} .
$$

Denote

$$
\Psi(G)_{\pi}=\{\chi \in \Psi(G) ; \chi \pi \cong \pi\} .
$$

Then $\Psi(G)_{\pi}$ is a finite group and $\chi \Psi(G)_{\pi} \mapsto \chi \pi$ is a one-to-one map from $\Psi(G) /$ $\Psi(G)_{\pi}$ onto $\Psi(G) \pi \subseteq \tilde{G}$.

Let $\Omega(G)$ be the set of all conjugacy classes of the pairs $(M, \rho)$, where $M$ is a Levi subgroup of $G$ and $\rho$ is an irreducible cuspidal representation of $M$. The sets

$$
\Omega=\{(M, \chi \rho): \chi \in \Psi(M)\} \subseteq \Omega(G)
$$

are called the connected components of $\Omega(G)$. A connected component will be called cuspidal if it contains a cuspidal pair $(M, \rho)$ such that $M=G$.

The mapping

$$
\chi \mapsto(M, \chi \rho), \quad \Psi(M) \rightarrow \Omega(G)
$$

has finite fibers (usually we shall not make any distinction between a pair $(M, \rho)$ and its conjugacy class in $\Omega(G)$ ). One defines the structure of a complex variety on $\Omega$ in a natural way (a function $f: \Omega(G) \rightarrow \mathbb{C}$ is called regular if the restriction of $f$ to any connected component of $\Omega(G)$ is regular). The algebra of all regular 
functions on $\Omega(G)$ will be denoted by $\mathfrak{Z}(G)$. For a subset $X \subseteq \Omega(G)$ we shall say that it is Zariski open if $X \cap \Omega$ is a Zariski open set in $\Omega$ for any connected component $\Omega$ of $\Omega(G)$. Zariski open sets in $\Omega(G)$ define a topology on $\Omega(G)$, which will be called the Zariski topology on $\Omega(G)$. Since connected components of $\Omega(G)$ are irreducible varieties, an open subset $O \subseteq \Omega(G)$ is dense (with respect to the Zariski topology) if and only if $O \cap \Omega \neq \emptyset$ for all connected components $\Omega$ of $\Omega(G)$. Further, the intersection of a finite number of open dense subsets in $\Omega(G)$ is an open dense subset of $\Omega(G)$.

Let $\pi \in \tilde{G}$. Then there is a parabolic subgroup $P=M N$ of $G$ and an irreducible cuspidal representation $\rho$ of $M$ such that $\pi$ is a subquotient of $\operatorname{Ind}_{P}^{G}(\rho)\left(\operatorname{Ind}_{P}^{G}(\rho)\right.$ denotes the representation of $G$ parabolically induced by $\rho$ from $P$; the induction that we consider is normalized). The class of $(\rho, M)$ in $\Omega(G)$ is uniquely determined by $\pi$. In this way we get a canonical projection

$$
\Pi: \pi \mapsto(M, \rho), \quad \tilde{G} \rightarrow \Omega(G) .
$$

The canonical projection has finite fibers.

We fix a Haar measure $d g$ on $G$. The convolution algebra of all compactly supported locally constant functions on $G$ will be denoted by $\mathcal{H}(G)$.

We shall recall some of the details of the Bernstein center in the rest of this section (see [BD] for more details).

Denote by $I$ the set of idempotents in $\mathcal{H}(G)$. Consider the order $\ll$ on $I: e \ll f$ if $e \in f * \mathcal{H}(G) * f$. For $e \in I$, denote by $Z(e * \mathcal{H}(G) * e)$ the center of the subalgebra $e * \mathcal{H}(G) * e$. One considers the family $Z(e * \mathcal{H}(G) * e), e \in I$, as a projective system, where the transition maps are given in the following way: if $e \ll f$, then the transition map $Z(f * \mathcal{H}(G) * f) \rightarrow Z(e * \mathcal{H}(G) * e)$ sends $z \mapsto e * z$. Denote the projective limit by $\mathcal{Z}(G)$. It consists of all systems of elements $h=(h(e))_{e \in I}$, $h(e) \in Z(e * \mathcal{H}(G) * e)$, which satisfy $h(e)=h(f) * e$ if $e \ll f$.

Let $(\pi, X)$ be a smooth representation of $G$, and let $h \in \mathcal{Z}(G), x \in X$. Take $e \in I$ such that $\pi(e) x=x$. Define $\pi(h) x$ to be $\pi(h(e)) x$. Then $\pi(h) x$ does not depend on $e$ as above. In this way we get a representation of the algebra $\mathcal{Z}(G)$ on $X$. The actions of $G$ and $\mathcal{Z}(G)$ commute, i.e. for each $h \in \mathcal{Z}(G)$ the mapping $\pi(h): X \rightarrow X$ is $G$-intertwining. Further, if $A: X_{1} \rightarrow X_{2}$ is an intertwining of two smooth representations of $G$, then it is also an intertwining of the corresponding representations of $\mathcal{Z}(G)$.

Suppose that $(\pi, X)$ is irreducible. Then $\mathcal{Z}(G)$ acts on $X$ by scalars which we shall denote by $\chi_{\pi}(h), h \in \mathcal{Z}(G)$. The character

$$
\chi_{\pi}
$$

of $\mathcal{Z}(G)$ that we obtain in this way is called the infinitesimal character of $\pi$.

Note that for each $h \in \mathcal{Z}(G)$ we obtain a function $h^{\prime}: \tilde{G} \rightarrow \mathbb{C}, \pi \mapsto \chi_{\pi}(h)$. This function factors through the canonical projection (3-1) $\Pi: \tilde{G} \rightarrow \Omega(G)$ by a function

$$
\hat{h}: \Omega(G) \rightarrow \mathbb{C} .
$$

Then $\hat{h} \in \mathfrak{Z}(G)$ and the mapping $h \mapsto \hat{h}$ is an isomorphism of $\mathcal{Z}(G)$ onto $\mathfrak{Z}(G)$.

Let $(\pi, X)$ be a smooth representation of $G$. We define a representation $\pi$ of $\mathfrak{Z}(G)$ on $X$ by $\pi(\hat{h}) x=\pi(h) x, h \in \mathcal{Z}(G), x \in X$. Clearly, $\mathfrak{Z}(G)$ acts by scalars in an irreducible representation $\pi$. The corresponding character of $\mathfrak{Z}(G)$ will again be denoted by $\chi_{\pi}$ and again called the infinitesimal character of $\pi$. Obviously, $\pi(\mathfrak{z}) x=\chi_{\pi}(\mathfrak{z}) x=\mathfrak{z}(\Pi(\pi)) x$ for $\mathfrak{z} \in \mathfrak{Z}(G), x \in X$. So, the infinitesimal character $\chi_{\pi}$ 
is given by evaluation of functions of $\mathfrak{Z}(G)$ in $\Pi(\pi)$. Therefore, there is a bijection between $\Omega(G)$ and the set of infinitesimal characters (which is given by evaluation of functions at points). This is the reason why we shall sometimes talk of $\Omega(G)$ as of the variety of infinitesimal characters of $G$.

We denote by $\mathfrak{Z}^{0}(G)$ the ideal of $\mathfrak{Z}(G)$, consisting of all functions supported on a finite number of components. An infinitesimal character $\omega$ can be interpreted as an algebra homomorphism

$$
\omega: \mathfrak{Z}(G) \rightarrow \mathbb{C},
$$

which is non-trivial on $\mathfrak{Z}^{0}(G)$.

We shall say that a smooth representation $(\pi, X)$ has an infinitesimal character if there exists $(M, \rho)$ such that $\pi(\mathfrak{z}) x=\mathfrak{z}((M, \rho)) x$ for $\mathfrak{z} \in \mathfrak{Z}(G), x \in X$.

For a function $\varphi \in \mathcal{H}(G)$, we denote by $\check{\varphi}$ a function defined by $\check{\varphi}(g)=\varphi\left(g^{-1}\right)$. Recall that $\left(\varphi_{1} * \varphi_{2}\right)^{-}=\check{\varphi}_{2} * \check{\varphi}_{1}$.

Let $h=(h(e))_{e \in I} \in \mathcal{Z}(G)$. Then $h(e) \in Z(e * \mathcal{H}(G) * e)$ and $h(e)=h(f) * e$ if $e \ll f$. Obviously $h(\check{e})^{\smile} \in Z(e * \mathcal{H}(G) * e)$. Note that $e \ll f$ if and only if $\check{e} \ll \check{f}$. Further, for $e \ll f$ we have $h(\check{e})^{\smile}=e * h(\check{f})^{\swarrow}=h(\check{f})^{\triangleleft} * e$. Therefore,

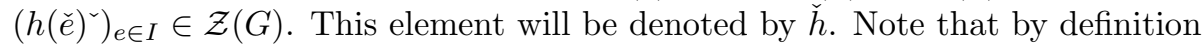

$$
\check{h}(e)=h(\check{e})^{\check{ }}, \quad h \in \mathcal{Z}(G) .
$$

From this follows $(\check{h})^{\swarrow}=h$ for $h \in \mathcal{Z}(G)$.

Let $(\pi, X)$ be a smooth representation of $G$ and let $Y$ be a finite-dimensional complex vector space. Take $A \in \operatorname{Hom}_{\mathbb{C}}(X, Y)^{(s)}, x \in X$ and $\varphi \in \mathcal{H}(G)$. Then

$$
\begin{aligned}
(L(\varphi) A)(x)= & \left(\int_{G} \varphi(g) L(g) A d g\right)(x)=\int_{G} \varphi(g)(L(g) A)(x) d g \\
= & \int_{G} \varphi(g) A\left(\pi\left(g^{-1}\right) x\right) d g=\int_{G} A\left(\varphi(g) \pi\left(g^{-1}\right) x\right) d g \\
= & \left.A\left(\int_{G} \varphi(g) \pi\left(g^{-1}\right) x d g\right)=A\left(\int_{G} \varphi\left(g^{-1}\right) \pi(g) x d g\right)\right)=A(\pi(\check{\varphi}) x .
\end{aligned}
$$

It follows that we have the following lemma.

3.1. Lemma. For a smooth representation $(\pi, X)$ of $G$, a finite-dimensional complex vector space $Y, A \in \operatorname{Hom}_{\mathbb{C}}(X, Y)^{(s)}, x \in X$ and $h \in \mathcal{Z}(G)$ we have

$$
(L(h) A)(x)=A(\pi(\check{h}) x) .
$$

In particular, $(\tilde{\pi}(h) \tilde{x})(x)=\tilde{x}(\pi(\check{h}) x)$ for $x \in X$ and $\tilde{x} \in \tilde{X}$.

Proof. Choose an open compact subgroup $H$ of $K$ such that $x$ and $A$ are invariant for the action of $H$. Denote by $e_{H}$ the characteristic function of $H$ divided by the Haar measure of $H$. Then $e_{H} \in I, \check{e}_{H}=e_{H}, L\left(e_{H}\right) A=A$ and $\pi\left(e_{H}\right) x=x$. Now

$$
\begin{aligned}
(L(h) A)(x)=\left(L\left(h\left(e_{H}\right)\right) A\right) & (x)=A\left(\pi\left(h\left(e_{H}\right)^{\smile}\right)\right)(x) \\
= & A\left(\pi\left(h\left(\check{e}_{H}\right)^{\smile}\right)\right)(x)=A\left(\pi\left(\check{h}\left(e_{H}\right)\right)(x)=A(\pi(\check{h})(x) .\right.
\end{aligned}
$$

This completes the proof.

The above lemma implies that for an irreducible representation $\pi$ we have

$$
\chi_{\pi}(h)=\chi_{\tilde{\pi}}(\check{h}), \quad h \in \mathcal{Z}(G) .
$$


For $\mathfrak{z} \in \mathfrak{Z}(G)$ we shall denote by $\tilde{\mathfrak{z}} \in \mathfrak{Z}(G)$ the function defined by

$$
\tilde{\mathfrak{z}}((M, \rho))=\mathfrak{z}((M, \tilde{\rho})) .
$$

3.2. Lemma. (i) For $h \in \mathcal{Z}(G)$ we have

$$
(\check{h})^{\wedge}=(\hat{h})^{\sim} \text {. }
$$

(ii) Let $(\pi, X)$ be a smooth representation of $G$ and let $Y$ be a finite-dimensional complex vector space. Then for $A \in \operatorname{Hom}_{\mathbb{C}}(X, Y)^{(s)}, x \in X$ and $\mathfrak{z} \in \mathfrak{Z}(G)$ we have

$$
(L(\mathfrak{z}) A)(x)=A(\pi(\tilde{\mathfrak{z}}) x) .
$$

Proof. (i) Let $\pi \in \tilde{G}$. Suppose that the image of $\pi$ under the canonical projection $\Pi: G \rightarrow \Omega(G)$ is $(M, \rho)$. Then $\tilde{\pi}$ goes to $(M, \tilde{\rho})$ under this projection. Using Lemma 3.1 we get

$$
\left.(\check{h})^{\wedge}((M, \rho))=\chi_{\pi}\left((\check{h})^{\wedge}\right)=\chi_{\pi}(\check{h})=\chi_{\tilde{\pi}}(h)=\hat{h}((M, \tilde{\rho}))=(\hat{h})^{\sim}(M, \rho)\right)
$$

(recall that $\mathcal{Z}(G) \cong \mathfrak{Z}(G)$ and that we consider infinitesimal characters as characters of $\mathcal{Z}(G)$ and of $\mathfrak{Z}(G)$, and denote them by the same symbol $\chi_{\pi}$; thus $\chi(h)=\chi_{\pi}(\hat{h})$ for $h \in \mathcal{Z}(G))$. This ends the proof of (i).

(ii) Let $\mathfrak{z}=\hat{h}$. Now from (i) we get

$$
(L(\mathfrak{z}) A)(x)=(L(h) A)(x)=A(\pi(\check{h}) x)=A\left(\pi\left((\check{h})^{\wedge}\right) x\right)=A\left(\pi\left((\hat{h})^{\sim}\right) x\right)=A(\pi(\tilde{\mathfrak{z}}) x) .
$$

Thus (ii) also holds.

Recall that we have considered the smooth representation $g \mapsto R_{g}$ (resp. $g \mapsto$ $L_{g}$ ) of $G$ on $C(G)^{(s, s)}$ given by the right (resp. left) translations of $G$. Since we denote by the same symbol the smooth representation and the corresponding representation of the Bernstein center, the representation $\mathfrak{z} \mapsto R_{\mathfrak{z}}$ (resp. $\mathfrak{z} \mapsto L_{\mathfrak{z}}$ ) of the Bernstein center will denote the representation corresponding to the smooth representation $g \mapsto R_{g}$ (resp. $g \mapsto L_{g}$ ) of $G$ on $C(G)^{(s, s)}$.

3.3. Lemma. Let $\mathfrak{z} \in \mathfrak{Z}(G)$ and $f \in C(G)^{(s, s)}$. Then

$$
R_{\mathfrak{z}} f=L_{\tilde{\mathfrak{z}}} f .
$$

Proof. Note that for $\psi \in \mathcal{H}(G)$ and $f \in C(G)^{(s, s)}$ we have

$$
L_{\psi} f=\psi * f \quad \text { and } \quad R_{\psi} f=f * \check{\psi} .
$$

Let $H$ be an open compact subgroup of $G$ such that $f$ is constant on double $H$-classes. For $\varphi \in Z\left(e_{H} * \mathcal{H}(G) * e_{H}\right)$ we shall show that $\varphi * f=f * \varphi$.

Fix $x \in G$. Introduce the function $f_{x}$ on $G$ :

$$
f_{x}(y)=\left\{\begin{array}{cc}
f(y) \text { for } y \in \operatorname{supp}(\varphi)^{-1} x H \cup H x \operatorname{supp}(\varphi)^{-1}, \\
0 \quad \text { for } y \notin \operatorname{supp}(\varphi)^{-1} x H \cup H x \operatorname{supp}(\varphi)^{-1} .
\end{array}\right.
$$

Obviously, $f_{x} \in e_{H} * \mathcal{H}(G) * e_{H}$. Recall $\varphi \in Z\left(e_{H} * \mathcal{H}(G) * e_{H}\right)$. Thus $\varphi * f_{x}=f_{x} * \varphi$ and we have

$$
\begin{aligned}
& (\varphi * f)(x)=\int_{G} \varphi(g) f\left(g^{-1} x\right) d g=\int_{G} \varphi(g) f_{x}\left(g^{-1} x\right) d g=\left(\varphi * f_{x}\right)(x) \\
& =\left(f_{x} * \varphi\right)(x)=\int_{G} f_{x}\left(x g^{-1}\right) \varphi(g) d g=\int_{G} f\left(x g^{-1}\right) \varphi(g) d g=(f * \varphi)(x) .
\end{aligned}
$$

Thus, $\varphi * f=f * \varphi$. 
Take $h \in \mathcal{Z}(G)$ such that $\mathfrak{z}=\hat{h}$. Now the above calculation implies

$$
\begin{aligned}
& L_{\mathfrak{z}} f=L_{\hat{h}} f=L_{h} f=L_{h\left(e_{H}\right)} f=h\left(e_{H}\right) * f=f * h\left(e_{H}\right)=f *\left(h\left(e_{H}\right)^{\sim}\right)^{\curlyvee} \\
& \quad=R_{h\left(e_{H}\right)^{\sim}} f=R_{h\left(\check{e}_{H}\right)^{\sim}} f=R_{\breve{h}\left(e_{H}\right)} f=R_{\breve{h}} f=R_{(\breve{h})^{\wedge}} f=R_{(\hat{h})^{\sim}} f=R_{\tilde{\mathfrak{z}}} f .
\end{aligned}
$$

3.4. Corollary. Let $V$ be a finite-dimensional complex vector space. Then for $z \in \mathfrak{Z}(G)$ and $\varphi \in C(G, V)^{(s, s)}$ we have $R_{\mathfrak{z}} \varphi=L_{\tilde{z}} \varphi$.

Proof. This follows from the fact that $C(G, V)^{(s, s)}$ and $C(G)^{(s, s)} \otimes V$ are isomorphic as $G \times G$-representations.

3.5. Remark. The above corollary implies that a subspace of $C(G, V)^{(s, s)}$ is invariant under the action of $\mathfrak{Z}(G)$ induced by the right translations if and only if it is invariant under the action of $\mathfrak{Z}(G)$ induced by the left translations.

Let us recall of the following result from $[\mathrm{BD}]$.

3.6. Theorem. Suppose that $(\pi, X)$ is a finitely generated smooth representation of $G$ and $H$ is an open compact subgroup of $G$. Then the space $X^{H}$ of all vectors in $X$ fixed by elements of $H$ is invariant for the action of $\mathfrak{Z}(G)$ and it is finitely generated as $\mathfrak{Z}^{0}(G)$-module.

The following immediate consequence of the above result will be useful for us.

3.7. Corollary. Each finitely generated smooth representation of $G$ which has an infinitesimal character is admissible. Moreover, it has finite length.

Let $M<G$ be a standard Levi subgroup. Define the morphism

$$
\mathrm{i}_{G M}: \Omega(M) \rightarrow \Omega(G)
$$

by $(L, \rho) \rightarrow(L, \rho)$. This is a finite morphism of algebraic varieties. The morphism $i_{G M}$ is not in general an inclusion, since cuspidal pairs conjugate under $G$ may be non-conjugate under $M$. We follow $[\mathrm{BDK}]$ and call the corresponding map

$$
\mathrm{i}_{G M}^{*}: \mathfrak{Z}(G) \rightarrow \mathfrak{Z}(M)
$$

the Harish-Chandra homomorphism. As for real groups, $\mathfrak{Z}(M)$ is a finitely generated $\mathfrak{Z}(G)$-module.

Let $P=M N$ be a parabolic subgroup of $G$. The functor of normalized parabolic induction was denoted by $\operatorname{Ind}_{P}^{G}$. The normalized Jacquet functor going in the opposite direction will be denoted by

$\mathrm{r}_{M G}$.

3.8. Proposition $([\overline{\mathrm{BD}}])$. Suppose that $\pi$ and $\sigma$ are smooth representations of $G$ and $M$, respectively. Let $\mathfrak{z} \in \mathfrak{Z}(G)$. Then

$$
\operatorname{Ind}_{P}^{G}\left(\sigma\left(\mathrm{i}_{G M}^{*}(\mathfrak{z})\right)\right)=\left(\operatorname{Ind}_{P}^{G}(\sigma)\right)(\mathfrak{z})
$$

$$
\mathrm{r}_{M G}(\pi(\mathfrak{z}))=\left(\mathrm{r}_{M G}(\pi)\right)\left(\mathrm{i}_{G M}^{*}(\mathfrak{z})\right) .
$$


Recall that $(M, \rho) \in \Omega(G)$ is called regular (in $G$ ) if there does not exist $w \in$ $G \backslash M$ which normalizes $M$ such that $\rho \cong w . \rho$, where $w . \rho$ denotes the representation $m \mapsto \rho\left(w^{-1} m w\right)$ of $M$.

Further, $(M, \rho) \in \Omega(G)$ will be called irreducible (in $G)$ if $\operatorname{Ind}_{P}^{G}(\rho)$ is irreducible.

We shall say that $(M, \rho) \in \Omega(G)$ is semi-simple in $G$ if each admissible representation $(\pi, X)$ of $G$ which has an infinitesimal character equal to $\mathfrak{z} \mapsto \mathfrak{z}((M, \rho))$ is semi-simple. Then also the corresponding infinitesimal character will be called semi-simple.

An infinitesimal character of $G$ will be called cuspidal if it corresponds to evaluation in an irreducible cuspidal representation of $G$ (i.e. to some $(G, \rho) \in \Omega(G)$ ).

3.9. Theorem. There exists an open dense subset $\Omega(G)^{\prime}$ of $\Omega(G)$ (with respect to the Zariski topology) such that each $(M, \rho) \in \Omega(G)^{\prime}$ is irreducible, regular and semi-simple.

We shall prove this claim in the eighth section (P. Schneider informed us that the claim of the proposition follows from Proposition 3.14 of $\mathrm{BD}$, but this proof seems to be quite a different type).

3.10. Remarks. (i) Note that Theorem 3.6 implies that each smooth representation with semi-simple infinitesimal character is semi-simple (see the proof of Lemma 8.1).

(ii) Each element $z$ which is in the center $Z(G)$ of $G$, defines in a natural way an element in $\mathcal{Z}(G)$ (one attaches $(z * e)_{e \in I} \in \mathcal{Z}(G)$ to $z$ ). This element will be denoted by

$$
z_{G} \text {. }
$$

One easily sees that for a smooth representation $\pi$ we have

$$
\pi\left(z_{G}\right)=\pi(z) .
$$

If in a smooth representation $\pi$ of $G$ each element of the center $Z(G)$ of $G$ acts by a scalar operator, then we shall say that $\pi$ has central character. The corresponding character of the center $Z(G)$ will be denoted by

$c_{\pi}$.

Observe that

$$
\left(z_{G}\right)^{\wedge}:(M, \rho) \mapsto c_{\operatorname{Ind}_{P}^{G}(\rho)}(z)=c_{\rho}(z) .
$$

(iii) We can also view the element $z_{G}$ from the above remark in the following way. By [BR], we can also define the Bernstein center as the center of the category of all the smooth representations of $G$ (i.e. the algebra of all the endomorphisms of the identity functor on the category of all the smooth representations of $G$ ). Now each $z \in Z(G)$ defines in an obvious way such an endomorphism $\pi \mapsto \pi(z) \in \operatorname{Hom}_{G}(\pi, \pi)$ of the category. This endomorphism corresponds to $z_{G}$.

(iv) One can easily see that (ii) implies that each smooth representation of $G$ with an infinitesimal character also has a central character (from interpretation of the Bernstein center in (iii) this is completely evident).

(v) Proposition 5.4.2 of [C] tells us that each admissible representation which is cuspidal and which has central character is semi-simple.

(vi) Note that (iv) and (v) imply that each $(M, \rho) \in \Omega(G)$ with

$$
M=G
$$

is semi-simple. 


\section{DeFinition of GENERALIZED SPHERICAL FUNCTIONS}

We retain the notation that $G$ is the group of rational points of a connected reductive group over a local non-archimedean field $F$. We fix a minimal parabolic subgroup $P_{\emptyset}$ of $G$. Subgroups of $G$ containing $P_{\emptyset}$ will be called standard parabolic subgroups. We fix a Levi factor $M_{\emptyset}$ of $P_{\emptyset}$. A Levi decomposition $P=M N$ of a standard parabolic subgroup $P$ of $G$ is called standard if $M_{\emptyset} \subseteq M$.

Denote by $A_{M}$ the maximal split torus in the center of $M$. Let $W_{M}=N_{G}\left(A_{M}\right) / M$ $=N_{G}(M) / Z_{G}\left(A_{M}\right)$, where $N_{G}\left(A_{M}\right)\left(\operatorname{resp} . Z_{G}\left(A_{M}\right)\right)$ denotes the normalizer (resp. the centralizer) of $A_{M}$ in $G$. We shall denote $W_{A_{\emptyset}}$ simply by $W$.

In the rest of this paper we shall fix a maximal compact subgroup $K$ of $G$ which satisfies Theorem 5 of [HC1] (such a subgroup always exists). Recall that $K$ is an open subgroup of $G$. In the sequel, we shall assume that the Haar measure of $G$ is normalized on $K$.

Of the properties required to hold for $K$ by this theorem, until Theorem 6.4 we shall need only that the Iwasawa decomposition

$$
G=K P_{\emptyset}
$$

holds (this is (i) of the theorem). For Theorem 6.4 we shall need the property that if $P=M N$ is a standard parabolic subgroup with the standard Levi decomposition, then

$$
K \cap P=(K \cap M)(K \cap N)
$$

(this is (iv) of Theorem 5 from [HC1]). For the seventh section we shall also need property $(\mathrm{v})$ of of the same theorem (this implies that if we fix a standard Levi subgroup $M$, the minimal parabolic subgroup $P_{\emptyset} \cap M$ in $M$, the Levi factor $M_{\emptyset}$ of $P_{\emptyset} \cap M$ and the maximal compact subgroup $K \cap M$ of $M$, then properties (4-1) and (4-2) hold also in this setting for $M$ ).

Let $\tau_{1}$ and $\tau_{2}$ be continuous finite-dimensional representations of $K$ on $V_{\tau_{1}}$ and $V_{\tau_{2}}$, respectively. Denote $\tau=\left(\tau_{1}, \tau_{2}\right)$ and let

$$
V=\operatorname{Hom}_{\mathbb{C}}\left(V_{\tau_{2}}, V_{\tau_{1}}\right) \text {. }
$$

We shall consider $(\tau, V)$ as a double representation of $K$ :

$$
\tau\left(k_{1}, k_{2}\right) \varphi=\tau_{1}\left(k_{1}\right) \varphi \tau_{2}\left(k_{2}\right)
$$

for $k_{1}, k_{2} \in K$ (it means that $\left(k_{1}, k_{2}\right) \mapsto \tau\left(k_{1}, k_{2}^{-1}\right)$ is a representation of $K \times K$ ).

Denote by $C(G, \tau)$ the space of all functions $\varphi: G \rightarrow V$ satisfying

$$
\varphi\left(k_{1} g k_{2}\right)=\tau_{1}\left(k_{1}\right) \varphi(g) \tau_{2}\left(k_{2}\right)
$$

for all $k_{1}, k_{2} \in K, g \in G$, i.e.

$$
L_{k_{1}^{-1}} R_{k_{2}} \varphi=\Lambda_{\tau_{1}\left(k_{1}\right) \circ} \Lambda_{\circ \tau_{2}\left(k_{2}\right)} \varphi
$$

where $\tau_{1}\left(k_{1}\right) \circ, \circ \tau_{2}\left(k_{2}\right): V \rightarrow V$ are the mappings $A \mapsto \tau_{1}\left(k_{1}\right) \circ A$ and $A \circ \tau_{2}\left(k_{2}\right)$, respectively. Clearly,

Set

$$
C(G, \tau) \subseteq C(G, V)^{(s, s)}
$$

$$
\mathcal{A}(G, \tau)=C(G, \tau) \cap \mathcal{A}(G, V)
$$

4.1. Lemma. The space $C(G, \tau)$ is invariant under action of $\mathfrak{Z}(G)$ with respect to both left and right action. 
Proof. To see this, take $\varphi \in C(G, V), \mathfrak{z} \in \mathfrak{Z}(G)$ and $k_{1}, k_{2} \in K$. Then using Lemma 3.3 we get

$L_{k_{1}^{-1}} R_{k_{2}}\left(L_{\mathfrak{z}} \varphi\right)=L_{\mathfrak{z}}\left(L_{k_{1}^{-1}} R_{k_{2}}(\varphi)\right)=L_{\mathfrak{z}}\left(\Lambda_{\tau_{1}\left(k_{1}\right) \circ} \Lambda_{\circ \tau_{2}\left(k_{2}\right)} \varphi\right)=\Lambda_{\tau_{1}\left(k_{1}\right) \circ} \Lambda_{\circ \tau_{2}\left(k_{2}\right)}\left(L_{\mathfrak{z}} \varphi\right)$.

Thus, $L_{\mathfrak{z}} \varphi \in C(G, \tau)$. In a similar way one shows that $R_{\mathfrak{z}} \varphi \in C(G, \tau)$.

Let $\omega$ be an infinitesimal character of $G$, i.e. a character of $\mathfrak{Z}(G)$ which is nontrivial on $\mathfrak{Z}^{0}(G)$. Denote

$$
\begin{aligned}
\mathcal{E}_{\omega}(G, V)= & \mathcal{E}_{\omega}^{L}(G, V)=\left\{\varphi \in C(G, V)^{(s, s)} ; L_{\mathfrak{z}} \varphi=\omega(\mathfrak{z}) \varphi \text { for } \mathfrak{z} \in \mathfrak{Z}(G)\right\}, \\
& \mathcal{E}_{\omega}^{R}(G, V)=\left\{\varphi \in C(G, V)^{(s, s)} ; R_{\mathfrak{z}} \varphi=\omega(\mathfrak{z}) \varphi \text { for } \mathfrak{z} \in \mathfrak{Z}(G)\right\} .
\end{aligned}
$$

4.2. Lemma. Each of the spaces $\mathcal{E}_{\omega}(G, V)$ and $\mathcal{E}_{\omega}^{R}(G, V)$ is invariant for the left and the right action of $G$.

Proof. Let $\varphi \in \mathcal{E}_{\omega}(G, V), g \in G$ and $\mathfrak{z} \in \mathfrak{Z}(G)$. Then $L_{\mathfrak{z}} L_{g} \varphi=L_{g} L_{\mathfrak{z}} \varphi=\omega(\mathfrak{z}) L_{g} \varphi$ and $L_{\mathfrak{z}} R_{g} \varphi=R_{g} L_{\mathfrak{z}} \varphi=\omega(\mathfrak{z}) R_{g} \varphi$. Thus, $L_{g} \varphi, R_{g} \varphi \in \mathcal{E}_{\omega}(G, V)$. In the same way one shows the invariance of $\mathcal{E}_{\omega}^{R}(G, V)$.

4.3. Corollary. The spaces $\mathcal{E}_{\omega}(G, V)$ and $\mathcal{E}_{\omega}^{R}(G, V)$ are contained in $\mathcal{A}(G, V)$.

Proof. By the above lemma, each $\varphi$ in $\mathcal{E}_{\omega}(G, V)$ (and in $\mathcal{E}_{\omega}^{R}(G, V)$ ) generates a (finitely generated) representation with an infinitesimal character. By Corollary 3.7 , it is admissible. Thus, $\varphi \in \mathcal{A}(G, V)$.

Denote

$$
\begin{aligned}
\mathcal{E}_{\omega}(G, \tau) & =\mathcal{E}_{\omega}(G, V) \cap C(G, \tau), \\
\mathcal{E}_{\omega}^{R}(G, \tau) & =\mathcal{E}_{\omega}^{R}(G, V) \cap C(G, \tau) .
\end{aligned}
$$

These spaces are contained in $\mathcal{A}(G, V)$ and $C(G, \tau)$. We shall denote

$$
\mathcal{A}(G, \tau)=\mathcal{A}(G, V) \cap C(G, \tau) .
$$

Then $\mathcal{E}_{\omega}(G, \tau)$ and $\mathcal{E}_{\omega}^{R}(G, \tau)$ are contained in $\mathcal{A}(G, \tau)$.

$$
\text { 5. The MAPPing } \varphi \mapsto T_{\varphi}
$$

We set

$$
C(G, V)_{\tau_{2}}^{(s)}=\left\{\varphi \in C(G, V)^{(s, s)} ; \varphi(g k)=\varphi(g) \tau_{2}(k) \text { for all } g \in G, k \in K\right\} .
$$

The above condition describing a $\phi$ in $C(G, V)_{\tau_{2}}^{(s)}$ can be expressed as

$$
R_{k} \varphi=\Lambda_{\circ \tau_{2}(k)} \varphi
$$

for all $k \in K$. Clearly, $C(G, V)_{\tau_{2}}^{(s)}$ is invariant for the left action of the group and the representation defined by this action is smooth. It follows that $C(G, V)_{\tau_{2}}^{(s)}$ is invariant for the left action of $\mathfrak{Z}(G))$. Therefore, it is also invariant under the right action of $\mathfrak{Z}(G)$ by Remark 3.5 (one can also see this directly from the fact that $R_{k} R_{\mathfrak{z}} \varphi=R_{\mathfrak{z}} R_{k} \varphi=R_{\mathfrak{z}} \Lambda_{\circ \tau_{2}(k)} \varphi=\Lambda_{\circ \tau_{2}(k)} R_{\mathfrak{z}} \varphi$ for $\mathfrak{z} \in \mathfrak{Z}(G), g \in G, k \in K$ and $\left.\varphi \in C(G, V)_{\tau_{2}}^{(s)}\right)$.

We shall look at the Hecke algebra of all locally constant compactly supported functions $\mathcal{H}(G)$ as a left $\mathcal{H}(G)$-module in an obvious way. This is the same action that we get if we look at the representation $L$ of $G$ on $\mathcal{H}(G)$ by left translations, and integrate it to the representation of the Hecke algebra $\mathcal{H}(G)$. 
We consider $\mathcal{H}(K) \subseteq \mathcal{H}(G)$ as a subalgebra. We shall consider $\mathcal{H}(G)$ as a right $\mathcal{H}(K)$-module in a natural way (the action of $\mathcal{H}(K)$ is given by the multiplication from the right-hand side; recall that the multiplication in $\mathcal{H}(G)$ is the convolution).

We shall consider the action of $G$ on $\mathcal{H}(G) \otimes_{\mathcal{H}(K)} V_{\tau_{1}}$ which comes from the action of $G$ on the first factor.

5.1. Lemma. The representation of $G$ on $\mathcal{H}(G) \otimes_{\mathcal{H}(K)} V_{\tau_{2}}$ is finitely generated.

Proof. Let $H$ be the kernel of $\tau_{2}$. Denote by $e_{H}$ the characteristic function of $\mathrm{H}$, divided by the measure of $H$. It is enough to show that $e_{H} \otimes V_{\tau_{2}}$ generates $\mathcal{H}(G) \otimes_{\mathcal{H}(K)} V_{\tau_{2}}$ as an $\mathcal{H}(G)$-module. Note that

$$
\begin{aligned}
\mathcal{H}(G) \otimes \otimes_{\mathcal{H}(K)} & V_{\tau_{2}}=\left\{h \otimes v ; h \in \mathcal{H}(G), v \in V_{\tau_{2}}\right\} \\
= & \left\{h \otimes e_{H} v ; h \in \mathcal{H}(G), v \in V_{\tau_{2}}\right\}=\left\{h * e_{H} \otimes v ; h \in \mathcal{H}(G), v \in V_{\tau_{2}}\right\} .
\end{aligned}
$$

This proves the lemma.

5.2. Lemma. Let $(\pi, X)$ be a smooth representation of $G$ and let $\omega$ be an infinitesimal character.

(i) The subspace $X_{[\omega]}$ spanned by

$$
\pi(\mathfrak{z}) x-\omega(\mathfrak{z}) x, \mathfrak{z} \in \mathfrak{Z}(G), x \in X,
$$

is $G$-invariant. The subrepresentation on this space will be denoted by

$$
\left(\pi_{[\omega]}, X_{[\omega]}\right) .
$$

(ii) The space $X / X_{[\omega]}$ will be denoted by $X^{\langle\omega\rangle}$. The quotient representation on this space will be denoted by

$$
\left(\pi^{\langle\omega\rangle}, X^{\langle\omega\rangle}\right)
$$

Then the representation $\left(\pi^{\langle\omega\rangle}, X^{\langle\omega\rangle}\right)$ has an infinitesimal character, and the infinitesimal character is $\omega$ (i.e. $\left(\pi^{\langle\omega\rangle}, X^{\langle\omega\rangle}\right)$ is an $\omega$-representation). If $\pi$ is finitely generated, then $\left(\pi^{\langle\omega\rangle}, X^{\langle\omega\rangle}\right)$ is admissible ( $\omega$-representation).

(iii) If $\mathfrak{Z}(G)$ acts on a complex vector space $W$, we shall denote

$$
W_{\omega}=\{w \in W ; \mathfrak{z} \cdot w=\omega(\mathfrak{z}) w \text { for all } \mathfrak{z} \in \mathfrak{Z}(G)\} .
$$

The space $X_{\omega}$ is $G$-invariant and the subrepresentation on this subspace will be denoted by

$$
\left(\pi_{\omega}, X_{\omega}\right)
$$

(iv) Let $U$ be a finite-dimensional complex vector space. There is a natural representation $L$ of $G$ on $\operatorname{Hom}_{\mathbb{C}}(X, U)$ (see section 2). The smooth part of this representation will be denoted by $\operatorname{Hom}_{\mathbb{C}}(X, U)^{(s)}$.

If $T: X \rightarrow U$ is a linear operator which vanishes on $X_{[\tilde{\omega}]}$, then $T$ factors through $X^{\langle\tilde{\omega}\rangle}=X / X_{[\tilde{\omega}]}$ by the operator which will be denoted by $T^{b}$. Then the mapping $T \mapsto T^{b}$ defines an isomorphism of $G$-representations

$$
\operatorname{Hom}_{\mathbb{C}}(X, U)_{\omega}^{(s)} \rightarrow \operatorname{Hom}_{\mathbb{C}}\left(X^{\langle\tilde{\omega}\rangle}, U\right)^{(s)} .
$$

Proof. The invariance in (i) and (iii) is clear.

To prove (ii), denote the quotient map by $\Phi$. Then

$$
\pi^{\langle\omega\rangle}(\mathfrak{z}) \Phi(x)-\omega(\mathfrak{z}) \Phi(x)=\Phi(\pi(\mathfrak{z}) x)-\omega(\mathfrak{z}) \Phi(x)=\Phi(\pi(\mathfrak{z}) x-\omega(\mathfrak{z}) x)=0 .
$$

The last claim of (ii) follows from Corollary 3.7. 
Now we shall prove (iv). Let $T \in \operatorname{Hom}_{\mathbb{C}}(X, U)_{\omega}^{(s)}$. Then $(L(\mathfrak{z}) T)(x)=\omega(\mathfrak{z}) T(x)$. Recall that $L(\mathfrak{z}) T(x)=T(\pi(\tilde{\mathfrak{z}}) x)$ by Lemma 3.1. Therefore

$$
T(\pi(\check{\mathfrak{z}}) x)=\omega(\mathfrak{z}) T(x)(=\tilde{\omega}(\check{\mathfrak{z}}) T(x))
$$

for all $\mathfrak{z} \in \mathfrak{Z}(G)$ and $x \in X$. Thus $T$ vanishes on all $\pi(\mathfrak{z}) x-\tilde{\omega}(\mathfrak{z}) x$. Therefore $T$ induces an operator in $\operatorname{Hom}_{\mathbb{C}}\left(X / X_{[\omega]}, U\right)$, which will be denoted by $T^{b}$. The mapping $T \mapsto T^{b}$, from $\operatorname{Hom}_{\mathbb{C}}(X, U)_{\omega}$ into $\operatorname{Hom}_{\mathbb{C}}\left(X / X_{[\tilde{\omega}]}, U\right)$, is obviously $G$-intertwining. Therefore, $T^{b}$ is in the smooth part $\operatorname{Hom}_{\mathbb{C}}\left(X / X_{[\tilde{\omega}]}, U\right)^{(s)}$ of $\operatorname{Hom}_{\mathbb{C}}\left(X^{\langle\tilde{\omega}\rangle}, U\right)=$ $\operatorname{Hom}_{\mathbb{C}}\left(X / X_{[\tilde{\omega}]}, U\right)$.

From the other side, $\operatorname{Hom}_{\mathbb{C}}\left(X / X_{[\tilde{\omega}]}, U\right)_{*}$ is a representation with an infinitesimal character $\omega$ (we see it in the same way as above). There is natural embedding $S \mapsto$ $S^{\sharp}$ of the space $\operatorname{Hom}_{\mathbb{C}}\left(X / X_{[\tilde{\omega}]}, U\right)^{(s)}$ into $\operatorname{Hom}_{\mathbb{C}}(X, U)_{\omega}^{(s)}$ (which is $G$-intertwining). Obviously, the mappings $T \mapsto T^{b}$ and $S \mapsto S^{\#}$ are inverses of each other. Therefore, we can identify $\operatorname{Hom}_{\mathbb{C}}(X, U)_{\omega}^{(s)}$ with $\operatorname{Hom}_{\mathbb{C}}\left(X / X_{[\tilde{\omega}]}, U\right)^{(s)}$ in this way.

We now define an action $L$ of $G$ on $\operatorname{Hom}_{\mathbb{C}}\left(\mathcal{H}(G) \otimes_{\mathcal{H}(K)} V_{\tau_{2}}, V_{\tau_{1}}\right)^{(s)}$ in a natural way:

$$
(L(g) A)(h \otimes v)=A\left(L_{g^{-1}} h \otimes v\right)
$$

(see section 2). Then for $f \in \mathcal{H}(G)$

$$
(L(f) A)(h \otimes v)=A(\check{f} * h \otimes v) .
$$

In this way we also have an action of $\mathfrak{Z}(G)$ on $\operatorname{Hom}_{\mathbb{C}}\left(\mathcal{H}(G) \otimes_{\mathcal{H}(K)} V_{\tau_{2}}, V_{\tau_{1}}\right)^{(s)}$.

Proposition 5.3. (i) Let $\varphi \in C(G, V)_{\tau_{2}}^{(s)}$. Then the formula

$$
\sum_{i} h_{i} \otimes v_{i} \mapsto \sum_{i}\left(\left(\check{h}_{i} * \varphi\right)(1)\right)\left(v_{i}\right)=\sum_{i}\left(\left(L_{\check{h}_{i}} \varphi\right)(1)\right)\left(v_{i}\right)
$$

defines a linear mapping

$$
T_{\varphi}: \mathcal{H}(G) \otimes_{\mathcal{H}(K)} V_{\tau_{2}} \rightarrow V_{\tau_{1}}
$$

(in particular, the above mapping is well defined).

(ii) The mapping

$$
\begin{aligned}
T: C(G, V)_{\tau_{2}}^{(s)} & \rightarrow H_{o m_{\mathbb{C}}}\left(\mathcal{H}(G) \otimes_{\mathcal{H}(K)} V_{\tau_{2}}, V_{\tau_{1}}\right)^{(s)}, \\
\varphi & \mapsto T_{\varphi}
\end{aligned}
$$

is an injective $G$-intertwining.

(iii) We have

$$
H_{o m_{\mathcal{H}(K)}}\left(\mathcal{H}(G) \otimes_{\mathcal{H}(K)} V_{\tau_{2}}, V_{\tau_{1}}\right) \subseteq H_{o m}\left(\mathcal{H}(G) \otimes_{\mathcal{H}(K)} V_{\tau_{2}}, V_{\tau_{1}}\right)^{(s)} .
$$

Further, $\operatorname{Hom}_{\mathcal{H}(K)}\left(\mathcal{H}(G) \otimes_{\mathcal{H}(K)} V_{\tau_{2}}, V_{\tau_{1}}\right)$ is invariant under the action of $\mathfrak{Z}(G)$.

(iv) If $\varphi \in C(G, \tau)$, then $T_{\varphi} \in H_{o m} m_{\mathcal{H}(K)}\left(\mathcal{H}(G) \otimes_{\mathcal{H}(K)} V_{\tau_{2}}, V_{\tau_{1}}\right)$.

Proof. (i) Let $\varphi \in C(G, V)_{\tau_{2}}^{(s)}$. Define

$$
T_{\varphi}^{\prime}: \mathcal{H}(G) \times V_{\tau_{2}} \rightarrow V_{\tau_{1}}, \quad(h, v) \mapsto((\check{h} * \varphi)(1))(v)=\left(\left(L_{\check{h}} \varphi\right)(1)\right)(v),
$$


where $\check{h}(x)=h\left(x^{-1}\right)\left(\right.$ recall $\left.\left(h_{1} * h_{2}\right)^{\swarrow}=\check{h}_{2} * \check{h}_{1}\right)$. Now for $b \in \mathcal{H}(K)$

$$
\begin{aligned}
& T_{\varphi}^{\prime}(h * b, v)=\left(\left(L_{(h * b)^{\sim}} \varphi\right)(1)\right)(v)=\left(\left(L_{\breve{b} * \check{h}} \varphi\right)(1)\right)(v)=\left(\left(L_{\breve{b}}\left(L_{\breve{h}} \varphi\right)\right)(1)\right)(v) \\
& =\int_{K} b(k) L_{k}\left(L_{\breve{h}} \varphi\right)(1) v d k=\int_{K} b(k)\left(L_{\breve{h}} \varphi\right)(k) v d k=\int_{K} b(k)\left(L_{\breve{h}} \varphi\right)(1) \tau_{2}(k) v d k \\
& =\left(L_{\check{h}} \varphi\right)(1) \int_{K} b(k) \tau_{2}(k) v d k=\left(L_{\check{h}} \varphi\right)(1) \tau_{2}(b) v=T_{\varphi}^{\prime}\left(h, \tau_{2}(b) v\right) .
\end{aligned}
$$

Therefore, $T_{\varphi}^{\prime}$ can be factored through

$T_{\varphi}: \mathcal{H}(G) \otimes_{\mathcal{H}(K)} V_{\tau_{2}} \rightarrow V_{\tau_{1}}, \quad \sum_{i} h_{i} \otimes v_{i} \mapsto \sum_{i}\left(\left(\check{h}_{i} * \varphi\right)(1)\right)\left(v_{i}\right)=\sum_{i}\left(\left(L_{\check{h}_{i}} \varphi\right)(1)\right)\left(v_{i}\right)$.

In this way we get that the mapping

$$
T: C(G, V)_{\tau_{2}}^{(s)} \rightarrow \operatorname{Hom}_{\mathbb{C}}\left(\mathcal{H}(G) \otimes_{\mathcal{H}(K)} V_{\tau_{2}}, V_{\tau_{1}}\right), \quad \varphi \mapsto T_{\varphi}
$$

is well defined. This mapping is obviously linear.

(ii) First we shall show that this mapping is injective. Suppose $T_{\varphi}=0$. Then

$$
0=T_{\varphi}(h \otimes v)=\left(\left(L_{\check{h}} \varphi\right)(1)\right)(v),
$$

for all $h \in \mathcal{H}(G)$ and $v \in V_{\tau_{2}}$. Thus

$$
\left(L_{h} \varphi\right)(1)=0 \text { for all } h \in \mathcal{H}(G) .
$$

Take $h \in \mathcal{H}(G)$ such that $L_{h} \varphi=\varphi$. Then for $x \in G$

$$
\begin{aligned}
\varphi(x)=\left(L_{h} \varphi\right)(x) & =\left(\int_{G} h(g)\left(L_{g} \varphi\right) d g\right)(x)=\int_{G} h(g) \varphi\left(g^{-1} x\right) d g \\
= & \int_{G} h(x g) \varphi\left(g^{-1}\right) d g=\int_{G}\left(L_{x^{-1}} h\right)(g) \varphi\left(g^{-1}\right) d g=\left(L_{L_{x^{-1}} h} \varphi\right)(1) .
\end{aligned}
$$

This implies $\varphi=0$. Thus, $T$ is injective.

Now take $\varphi \in C(G, V)_{\tau_{2}}^{(s)}$. Then for $f \in \mathcal{H}(G)$

$$
\begin{aligned}
T_{L_{f} \varphi}(h \otimes v)=T_{f * \varphi}(h \otimes v) & =(\check{h} * f * \varphi)(1) v \\
& =T_{\varphi}(\check{f} * h \otimes v)=T_{\varphi}\left(L_{\breve{f}} h \otimes v\right)=\left(L(f) T_{\varphi}\right)(h \otimes v) .
\end{aligned}
$$

This shows that $\varphi \mapsto T_{\varphi}$ is $G$-intertwining.

Since $T$ is $G$-intertwining, the image of $T$ is in the smooth part

$$
\operatorname{Hom}_{\mathbb{C}}\left(\mathcal{H}(G) \otimes_{\mathcal{H}(K)} V_{\tau_{2}}, V_{\tau_{1}}\right)^{(s)}
$$

of $\operatorname{Hom}_{\mathbb{C}}\left(\mathcal{H}(G) \otimes_{\mathcal{H}(K)} V_{\tau_{2}}, V_{\tau_{1}}\right)$.

(iii) The inclusion in (iii) follows directly from the definition of

$$
\operatorname{Hom}_{\mathcal{H}(K)}\left(\mathcal{H}(G) \otimes_{\mathcal{H}(K)} V_{\tau_{2}}, V_{\tau_{1}}\right) \text {. }
$$

Let $B$ be a linear operator on $V_{\tau_{1}}$. Then the operator

$$
\Lambda_{B \circ}: \operatorname{Hom}_{\mathbb{C}}\left(\mathcal{H}(G) \otimes_{\mathcal{H}(K)} V_{\tau_{2}}, V_{\tau_{1}}\right) \rightarrow \operatorname{Hom}_{\mathbb{C}}\left(\mathcal{H}(G) \otimes_{\mathcal{H}(K)} V_{\tau_{2}}, V_{\tau_{1}}\right), A \mapsto B A
$$

is $G$-intertwining since

$$
\begin{aligned}
\left(L(g)\left(\Lambda_{B \circ} A\right)\right)(h \otimes v)=\left(\Lambda_{B \circ} A\right) & \left(L_{g^{-1}} h \otimes v\right)=B\left(A\left(L_{g^{-1}} h \otimes v\right)\right) \\
& =B((L(g) A)(h \otimes v))=\left(\Lambda_{B \circ}(L(g) A)\right)(h \otimes v) .
\end{aligned}
$$


We shall now show that $L(\mathfrak{z}) A \in \operatorname{Hom}_{\mathcal{H}(K)}\left(\mathcal{H}(G) \otimes_{\mathcal{H}(K)} V_{\tau_{2}}, V_{\tau_{1}}\right)$ if $A$ is in the space $\operatorname{Hom}_{\mathcal{H}(K)}\left(\mathcal{H}(G) \otimes_{\mathcal{H}(K)} V_{\tau_{2}}, V_{\tau_{1}}\right)$ and $\mathfrak{z} \in \mathfrak{Z}(G)$. To see this, take $k \in K$. First

$$
(L(\mathfrak{z}) A)\left(L_{k} h \otimes v\right)=\left(L\left(k^{-1}\right)(L(\mathfrak{z}) A)\right)(h \otimes v)
$$

by the definition (5-1) of the action of $K$ (and $G$ ). Recall that $\mathfrak{z} \mapsto L(\mathfrak{Z})$ is the action of the Bernstein center corresponding to the representation $g \mapsto L(g)$ of $G$ (defined by (5-1)). Therefore, these two actions commute $(L(\mathfrak{z})$ 's are intertwinings) and we have $L\left(k^{-1}\right)(L(\mathfrak{z}) A)=L(\mathfrak{z}) L\left(k^{-1}\right) A$. Therefore

$$
\begin{aligned}
& (L(\mathfrak{z}) A)\left(L_{k} h \otimes v\right)=\left(L(\mathfrak{z}) L\left(k^{-1}\right) A\right)(h \otimes v) \\
& \quad=L(\mathfrak{z})\left(L\left(k^{-1}\right) A(h \otimes v)\right)=L(\mathfrak{z})\left(A\left(L_{k} h \otimes v\right)\right)=L(\mathfrak{z})\left(\tau_{1}(k)(A(h \otimes v))\right) \\
& \quad=L(\mathfrak{z})\left(\Lambda_{\tau_{1}(k)} A\right)(h \otimes v)=\Lambda_{\tau_{1}(k)} L(\mathfrak{z}) A(h \otimes v)=\tau_{1}(k)((L(\mathfrak{z}) A)(h \otimes v)) .
\end{aligned}
$$

This shows that $\operatorname{Hom}_{\mathcal{H}(K)}\left(\mathcal{H}(G) \otimes_{\mathcal{H}(K)} V_{\tau_{2}}, V_{\tau_{1}}\right)$ is invariant for the action of $\mathfrak{Z}(G)$.

(iv) Let $\varphi \in C(G, \tau)$. For $h \in \mathcal{H}(G), v \in V_{\tau_{2}}$ and $k \in K$ we have

$$
\begin{aligned}
& T_{\varphi}\left(L_{k} h \otimes v\right)=\left(\left(\left(L_{k} h\right)^{\triangleleft} * \varphi\right)(1)\right) v=\int_{G} L_{k} h(g) \varphi(g) v d g=\int_{G} h\left(k^{-1} g\right) \varphi(g) v d g \\
= & \int_{G} h(g) \varphi(k g) v d g=\tau_{1}(k) \int_{G} h(g) \varphi(g) v d g=\tau_{1}(k)(\check{h} * \varphi)(1) v=\tau_{1}(k) T_{\varphi}(h \otimes v) .
\end{aligned}
$$

This shows $T_{\varphi} \in \operatorname{Hom}_{\mathcal{H}(K)}\left(\mathcal{H}(G) \otimes_{\mathcal{H}(K)} V_{\tau_{2}}, V_{\tau_{1}}\right)$.

5.4. Proposition. There exists a one-to-one linear mapping

$$
\mathcal{E}_{\omega}(G, \tau) \quad \rightarrow \quad \operatorname{Hom}_{\mathcal{H}(K)}\left(\left(\mathcal{H}(G) \otimes_{\mathcal{H}(K)} V_{\tau_{2}}\right)^{\langle\tilde{\omega}\rangle}, V_{\tau_{1}}\right) .
$$

Proof. Recall that for $\varphi \in C(G, \tau)$, by (iv) of Proposition 5.3 we have

$$
T_{\varphi} \in \operatorname{Hom}_{\mathcal{H}(K)}\left(\mathcal{H}(G) \otimes_{\mathcal{H}(K)} V_{\tau_{2}}, V_{\tau_{1}}\right) .
$$

Further, for $\varphi \in \mathcal{E}_{\omega}(G, \tau)$ by (ii) of the same proposition, $T_{\varphi}$ needs to be in

$$
\begin{aligned}
\operatorname{Hom}_{\mathbb{C}}\left(\mathcal{H}(G) \otimes_{\mathcal{H}(K)} V_{\tau_{2}}, V_{\tau_{1}}\right)_{\omega}^{(s)} \cap \operatorname{Hom}_{\mathcal{H}(K)}\left(\mathcal{H}(G) \otimes_{\mathcal{H}(K)} V_{\tau_{2}}, V_{\tau_{1}}\right) \\
=\operatorname{Hom}_{\mathcal{H}(K)}\left(\mathcal{H}(G) \otimes_{\mathcal{H}(K)} V_{\tau_{2}}, V_{\tau_{1}}\right)_{\omega} .
\end{aligned}
$$

We also know that $\varphi \mapsto T_{\varphi}$ is a one-to-one mapping. Therefore, we have a one-to one mapping from $\mathcal{E}_{\omega}(G, \tau)$ into $\operatorname{Hom}_{\mathcal{H}(K)}\left(\mathcal{H}(G) \otimes_{\mathcal{H}(K)} V_{\tau_{2}}, V_{\tau_{1}}\right)_{\omega}$. This one-to-one mapping goes from $\mathcal{E}_{\omega}(G, \tau)$ into

$$
\begin{aligned}
\operatorname{Hom}_{\mathcal{H}(K)}\left(\mathcal{H}(G) \otimes_{\mathcal{H}(K)} V_{\tau_{2}}, V_{\tau_{1}}\right)_{\omega} & \\
=\operatorname{Hom}_{\mathbb{C}}\left(\mathcal{H}(G) \otimes_{\mathcal{H}(K)} V_{\tau_{2}}, V_{\tau_{1}}\right)_{\omega}^{(s)} & \cap \operatorname{Hom}_{\mathcal{H}(K)}\left(\mathcal{H}(G) \otimes_{\mathcal{H}(K)} V_{\tau_{2}}, V_{\tau_{1}}\right) \\
\cong \operatorname{Hom}_{\mathbb{C}}\left(\left(\mathcal{H}(G) \otimes_{\mathcal{H}(K)} V_{\tau_{2}}\right)^{\langle\tilde{\omega}\rangle}, V_{\tau_{1}}\right)^{(s)} & \cap \operatorname{Hom}_{\mathcal{H}(K)}\left(\left(\mathcal{H}(G) \otimes_{\mathcal{H}(K)} V_{\tau_{2}}\right)^{\langle\tilde{\omega}\rangle}, V_{\tau_{1}}\right) \\
& =\operatorname{Hom}_{\mathcal{H}(K)}\left(\left(\mathcal{H}(G) \otimes_{\mathcal{H}(K)} V_{\tau_{2}}\right)^{\langle\tilde{\omega}\rangle}, V_{\tau_{1}}\right) .
\end{aligned}
$$

This proves the proposition.

5.5. Corollary. The spaces of generalized spherical functions $\mathcal{E}_{\omega}(G, \tau)$ are finite dimensional.

Proof. Note that $\left(\mathcal{H}(G) \otimes_{\mathcal{H}(K)} V_{\tau_{2}}\right)^{\langle\tilde{\omega}\rangle}$ is an admissible representation by Corollary 3.7, Lemma 5.1 and Lemma 5.2. This and the above proposition imply the corollary. 
6. The MAPPING $T \mapsto \varphi_{T}$

For $T \in \operatorname{Hom}_{\mathbb{C}}\left(\mathcal{H}(G) \otimes_{\mathcal{H}(K)} V_{\tau_{2}}, V_{\tau_{1}}\right)^{(s)}$ we define $\varphi_{T}: G \rightarrow V$ by the formula

$$
\varphi_{T}(g)(v)=T\left(L_{g}\left(e_{K_{0}} \otimes v\right)\right), \quad v \in V_{\tau_{2}}
$$

where $L$ denotes the natural representation of $G$ on $\mathcal{H}(G) \otimes V$ (the action is on the first factor), and

$$
K_{0}=\operatorname{Ker}\left(\tau_{1}\right) \cap \operatorname{Ker}\left(\tau_{2}\right) .
$$

6.1. Proposition. (i) The mapping $T \mapsto \varphi_{T}$ is a one-to-one $G$-intertwining from

$$
H_{o m}\left(\mathcal{H}(G) \otimes_{\mathcal{H}(K)} V_{\tau_{2}}, V_{\tau_{1}}\right)^{(s)} \text { into } C(G, V)_{\tau_{2}}^{(s)} \text {. }
$$

(ii) There exists a one-to-one $G$-intertwining from

$$
H_{\mathrm{om}}\left(\mathcal{H}(G) \otimes_{\mathcal{H}(K)} V_{\tau_{2}}, V_{\tau_{1}}\right)_{\omega}^{(s)} \cong \operatorname{Hom}_{\mathbb{C}}\left(\left(\mathcal{H}(G) \otimes_{\mathcal{H}(K)} V_{\tau_{2}}\right)^{\langle\tilde{\omega}\rangle}, V_{\tau_{1}}\right)^{(s)}
$$

into

$$
C(G, V)_{\tau_{2}}^{(s)} \cap \mathcal{E}_{\omega}(G, V) .
$$

(iii) If $T \in \operatorname{Hom}_{\mathcal{H}(K)}\left(\mathcal{H}(G) \otimes_{\mathcal{H}(K)} V_{\tau_{2}}, V_{\tau_{1}}\right)$, then $\varphi_{T} \in C(G, \tau)$.

(iv) There exists a one-to-one linear mapping of

$$
\operatorname{Hom}_{\mathcal{H}(K)}\left(\left(\mathcal{H}(G) \otimes_{\mathcal{H}(K)} V_{\tau_{2}}\right)^{\langle\tilde{\omega}\rangle}, V_{\tau_{1}}\right) \text { into } \mathcal{E}_{\omega}(G, \tau) .
$$

Proof. (i) For $k \in K$ and $\varphi \in \operatorname{Hom}_{\mathbb{C}}\left(\mathcal{H}(G) \otimes_{\mathcal{H}(K)} V_{\tau_{2}}, V_{\tau_{1}}\right)^{(s)}$ we have

$$
\begin{aligned}
\varphi_{T}(g k)(v) & \left.=T\left(L_{g k}\left(e_{K_{0}} \otimes v\right)\right)=T\left(L_{g k} e_{K_{0}} \otimes v\right)\right)=T\left(L_{g} L_{k} e_{K_{0}} \otimes v\right) \\
& =T\left(L_{g}\left(L_{k} e_{K_{0}} \otimes v\right)\right)=T\left(L_{g}\left(\frac{1}{\mu\left(K_{0}\right)} \operatorname{ch}_{k K_{0}} \otimes v\right)\right) \\
& =T\left(L_{g}\left(e_{K_{0}} \otimes \tau_{2}\left(\frac{1}{\mu\left(K_{0}\right)} \operatorname{ch}_{k K_{0}}\right) v\right)\right)=T\left(L_{g}\left(e_{K_{0}} \otimes \tau_{2}(k) v\right)\right) \varphi_{T}(g)\left(\tau_{2}(k) v\right)
\end{aligned}
$$

(we denoted in the above computation the characteristic function of a subset $Y$ by $\left.\operatorname{ch}_{Y}\right)$. This implies that $\varphi_{T} \in C(G, V)_{\tau_{2}}$.

We shall now show that $T \mapsto \varphi_{T}$ is a $G$-intertwining:

$$
\begin{aligned}
\left(L_{x} \varphi_{T}\right)(g) v=\varphi_{T}\left(x^{-1} g\right) v & =T\left(L_{x^{-1}}\left(e_{K_{0}} \otimes v\right)\right)=T\left(\left(L_{x^{-1}} e_{K_{0}}\right) \otimes v\right) \\
& \left.=T\left(L_{x^{-1}}\left(L_{g} e_{K_{0}}\right) \otimes v\right)=L(x) T\left(L_{g}\left(e_{K_{0}}\right) \otimes v\right)\right) \\
& \left.=(L(x) T)\left(L_{g}\left(e_{K_{0}}\right) \otimes v\right)\right)=\varphi_{L(x) T}(g) v .
\end{aligned}
$$

Note that now we also get that $\varphi_{T} \in C(G, V)_{\tau_{2}}^{(s)}$.

Now we shall prove that $T \mapsto \varphi_{T}$ is injective. Suppose

$$
T \in \operatorname{Hom}_{\mathbb{C}}\left(\mathcal{H}(G) \otimes_{\mathcal{H}(K)} V_{\tau_{2}}, V_{\tau_{1}}\right)^{(s)}
$$

and $\varphi_{T}(g) v=0$ for all $g \in G$ and $v \in V_{\tau_{2}}$. Then $T\left(L_{g}\left(e_{K_{0}} \otimes v\right)\right)=T\left(L_{g} e_{K_{0}} \otimes v\right)=0$. Since the set of $L_{g} e_{K_{0}} \otimes v$ generate vector space $\mathcal{H}(G) \otimes_{\mathcal{H}(K)} V_{\tau_{2}}$, we have $T=0$.

(ii) This follows directly from (i).

(iii) Suppose that $\varphi \in \operatorname{Hom}_{\mathcal{H}(K)}\left(\mathcal{H}(G) \otimes_{\mathcal{H}(K)} V_{\tau_{2}}, V_{\tau_{1}}\right)$. Then we have for $k \in K$ :

$$
\varphi_{T}(k g)(v)=T\left(L_{k g}\left(e_{K_{0}} \otimes v\right)\right)=\tau_{1}(k) T\left(L_{g}\left(e_{K_{0}} \otimes v\right)\right)=\tau_{1}(k) \varphi_{T}(g) v .
$$

Thus, $\varphi_{T} \in C(G, \tau)$.

(iv) We get (iv) directly from (i) and (iii). 
6.2. Remarks. (i) The study of generalized spherical functions in a natural way reduces to the case of $\tau_{1}$ and $\tau_{2}$ irreducible representations of $K$. For a smooth representation $(\pi, X)$ of $G$, we shall denote by

$$
X\left[\tau_{1}\right]
$$

the $\tau_{1}$-isotipic subspace of $X$.

(ii) Note that

$$
\begin{aligned}
e_{K_{0}} \otimes: & V_{\tau_{2}} \rightarrow \mathcal{H}(G) \otimes \mathcal{H}(K) V_{\tau_{2}}, \\
& v \mapsto e_{K_{0}} \otimes v,
\end{aligned}
$$

which shows up in the definition of $T \mapsto \varphi_{T}$ on $\operatorname{Hom}_{\mathbb{C}}\left(\mathcal{H}(G) \otimes_{\mathcal{H}(K)} V_{\tau_{2}}, V_{\tau_{1}}\right)^{(s)}$, is a $K$-intertwining. Therefore, if $\tau_{2}$ is irreducible, then $e_{K_{0}} \otimes v \in\left(\mathcal{H}(G) \otimes_{\mathcal{H}(K)} V_{\tau_{2}}\right)\left[\tau_{2}\right]$ for $v \in V_{\tau_{2}}$.

(iii) If we consider the mapping that $T \mapsto \varphi_{T}$ defines on

$$
\operatorname{Hom}_{\mathbb{C}}\left(\left(\mathcal{H}(G) \otimes_{\mathcal{H}(K)} V_{\tau_{2}}\right)^{\langle\tilde{\omega}\rangle}, V_{\tau_{1}}\right)^{(s)}
$$

(which is isomorphic to $\left.\operatorname{Hom}_{\mathbb{C}}\left(\mathcal{H}(G) \otimes_{\mathcal{H}(K)} V_{\tau_{2}}, V_{\tau_{1}}\right)_{\omega}^{(s)}\right)$, it is given by the formula

$$
g \mapsto\left(v \mapsto T\left(L_{g}\left(e_{K_{0}} \otimes v\right)\right)+\left(\mathcal{H}(G) \otimes_{\mathcal{H}(K)} V_{\tau_{2}}\right)_{[\tilde{\omega}]}\right) .
$$

Note again that

$$
v \mapsto e_{K_{0}} \otimes v+\left(\mathcal{H}(G) \otimes_{\mathcal{H}(K)} V_{\tau_{2}}\right)_{[\tilde{\omega}]}
$$

is a $K$-intertwining of $V_{\tau_{2}}$ (now into $\left(\mathcal{H}(G) \otimes_{\mathcal{H}(K)} V_{\tau_{2}}\right)^{\langle\tilde{\omega}\rangle}$ ). This $K$-intertwining will be denoted by

$$
\bar{e}_{K_{0}} \otimes .
$$

(iv) Let the infinitesimal character $\omega$ correspond to $(M, \rho)$ and assume that $\tau_{2}$ is irreducible.

Now we shall show that $\tau_{2}$ is a $K$-type of $\operatorname{Ind}_{P}^{G}(\tilde{\rho})$ if and only if

$$
\left(\mathcal{H}(G) \otimes_{\mathcal{H}(K)} V_{\tau_{2}}\right)^{\langle\tilde{\omega}\rangle} \neq\{0\} .
$$

Suppose first that $\tau_{2}$ is a $K$-type of $\operatorname{Ind}_{P}^{G}(\tilde{\rho})$. Then there exists a non-zero $K$-intertwining $\mu: V_{\tau_{2}} \rightarrow \operatorname{Ind}_{P}^{G}(\tilde{\rho})$. Consider the mapping

$$
(f, v) \mapsto R_{f}(\mu(v)), \quad \mathcal{H}(G) \times V_{\tau_{2}} \rightarrow \operatorname{Ind}_{P}^{G}(\tilde{\rho}) .
$$

This is obviously a non-trivial bilinear mapping. Consider now for $f \in \mathcal{H}(G), h \in$ $\mathcal{H}(K)$ and $v \in V_{\tau_{2}}$

$$
R_{f * h}(\mu(v))=R_{f} R_{h}(\mu(v))=R_{f}\left(\mu\left(\tau_{2}(h) v\right)\right) .
$$

This implies that $\left(\mathcal{H}(G) \otimes_{\mathcal{H}(K)} V_{\tau_{2}}\right)^{\langle\tilde{\omega}\rangle} \neq\{0\}$.

Suppose now that $\left(\mathcal{H}(G) \otimes_{\mathcal{H}(K)} V_{\tau_{2}}\right)^{\langle\tilde{\omega}\rangle} \neq\{0\}$. Then there exists a non-trivial $G$-intertwining

$$
I: \mathcal{H}(G) \otimes_{\mathcal{H}(K)} V_{\tau_{2}} \rightarrow X
$$

where $X$ is a (non-zero) subquotient of $\operatorname{Ind}_{P}^{G}(\tilde{\rho})$ (the restriction to $X$ of the quotient action of $G$ on $\operatorname{Ind}_{P}^{G}(\tilde{\rho})$ will be denoted by $\left.R^{X}\right)$. Since $I \neq 0$, one sees directly that $I\left(e_{K_{0}} \otimes v\right) \neq 0$ for some $v \in V_{\tau_{2}}$. Now consider the non-trivial linear mapping

$$
I_{e_{K_{0}}}: v \mapsto I\left(e_{K_{0}} \otimes v\right), \quad V_{\tau_{2}} \rightarrow X .
$$


Note that for $h \in \mathcal{H}(K)$ we have

$$
\begin{aligned}
I_{e_{K_{0}}}\left(\tau_{2}(h) v\right)=I\left(e_{K_{0}} \otimes \tau_{2}(h) v\right) & =I\left(e_{K_{0}} * h \otimes v\right) \\
= & I\left(h * e_{K_{0}} \otimes v\right)=R_{h}^{X} I\left(e_{K_{0}} \otimes v\right)=R_{h}^{X} I_{e_{K_{0}}}(v)
\end{aligned}
$$

(above we have used that $e_{K_{0}} * h=h * e_{K_{0}}$, which holds since $K_{0}$ is a normal subgroup of $K)$. Thus, we have shown that $\tau_{2}$ is a $K$-type of $\operatorname{Ind}_{P}^{G}(\tilde{\rho})$.

(v) Observe that there exists an injective $K$-intertwining of $X$ into $\operatorname{Ind}_{P}^{G}(\tilde{\rho})$. From this one can easily see that in (iv) we have proved for not necessarily irreducible $\tau_{2}$ that

$$
\left(\mathcal{H}(G) \otimes_{\mathcal{H}(K)} V_{\tau_{2}}\right)^{\langle\tilde{\omega}\rangle} \neq\{0\} \Longleftrightarrow \operatorname{Hom}_{K}\left(V_{\tau_{2}}, \operatorname{Ind}_{P}^{G}(\tilde{\rho})\right) \neq\{0\} .
$$

Now we shall consider a slightly modified setting. Let $(\pi, X)$ be a smooth representation of $G$ with infinitesimal character $\tilde{\omega}$. Consider the natural representation of $G$ on $\operatorname{Hom}_{\mathbb{C}}\left(X, V_{\tau_{1}}\right)$ (recall that $\operatorname{Hom}_{\mathbb{C}}\left(X, V_{\tau_{1}}\right)^{(s)}$ denotes the smooth part of it). Fix $\alpha \in \operatorname{Hom}_{K}\left(V_{\tau_{2}}, X\right)$. For $T \in \operatorname{Hom}_{\mathbb{C}}\left(X, V_{\tau_{1}}\right)^{(s)}$ define $\varphi_{T}^{\alpha}: G \rightarrow V$ by

$$
\varphi_{T}^{\alpha}(g) v=T(\pi(g)(\alpha(v))), v \in V_{\tau_{2}}, g \in G .
$$

Further, $T \mapsto \varphi_{T}^{\alpha}$ is a linear mapping from $\operatorname{Hom}_{\mathbb{C}}\left(X, V_{\tau_{1}}\right)^{(s)}$ into $C(G, V)_{\tau_{2}}$. Since it is a $G$-intertwining, the image is in $C(G, V)_{\tau_{2}}^{(s)}$. Further, if $T \in \operatorname{Hom}_{K}\left(X, V_{\tau_{1}}\right)$, then $\varphi_{T}^{\alpha} \in C(G, \tau)$. Thus, $\varphi_{T}^{\alpha} \in \mathcal{E}_{\omega}(G, V)$ (since $\pi$ has infinitesimal character $\tilde{\omega}$ ). In this way we obtain a linear mapping

$$
\begin{aligned}
\Phi_{X}: \operatorname{Hom}_{K}\left(V_{\tau_{2}}, X\right) \otimes \operatorname{Hom}_{K}\left(X, V_{\tau_{1}}\right) & \rightarrow \mathcal{E}_{\omega}(G, \tau), \\
\alpha \otimes T & \mapsto \varphi_{T}^{\alpha} .
\end{aligned}
$$

6.3. Lemma. (i) If $X=X_{1} \oplus X_{2}$ is a sum of $G$-subrepresentations, then $\operatorname{Im}\left(\Phi_{X_{1}}\right)+$ $\operatorname{Im}\left(\Phi_{X_{2}}\right)=\operatorname{Im}\left(\Phi_{X}\right)$.

(ii) If $\pi$ is irreducible, then $\Phi_{X}$ is injective.

(iii) If $\left(\pi^{\prime}, X^{\prime}\right)$ is a smooth representation of $G$ isomorphic to $(\pi, X)$, then

$$
\operatorname{Im}\left(\Phi_{X}\right)=\operatorname{Im}\left(\Phi_{X^{\prime}}\right) .
$$

Proof. (i) One gets $\operatorname{Im}\left(\Phi_{X_{i}}\right) \subseteq \operatorname{Im}\left(\Phi_{X}\right)$ directly (if $\varphi_{T}^{\alpha} \in \operatorname{Im}\left(\Phi_{X_{i}}\right)$, then extend the codomain of $\alpha$ and the domain of $T$ in the obvious way).

Denote the action of $G$ in $X_{i}$ by $\pi_{i}$. Let $\varphi_{T}^{\alpha} \in \operatorname{Im}\left(\Phi_{X}\right)$. Let $\alpha_{i}$ be the composition $V_{\tau_{2}} \stackrel{\alpha}{\rightarrow} X_{1} \oplus X_{2} \stackrel{\mathrm{pr}_{i}}{\rightarrow} X_{i}$ and $T_{i}$ the composition $X_{i} \hookrightarrow X_{1} \oplus X_{2} \stackrel{T}{\rightarrow} X_{1} \oplus X_{2} \stackrel{\mathrm{pr}_{i}}{\rightarrow} X_{i}$. Now

$$
\begin{aligned}
\varphi_{T}^{\alpha}(g) v & =T(\pi(g)(\alpha(v)))=T\left(\pi(g)\left(\alpha_{1}(v)+\alpha_{2}(v)\right)\right) \\
& =T\left(\pi_{1}(g)\left(\alpha_{1}(v)\right)+\pi_{2}(g)\left(\alpha_{2}(v)\right)\right) \\
& =T_{1}\left(\pi_{1}(g)\left(\alpha_{1}(v)\right)\right)+T_{2}\left(\pi_{2}(g)\left(\alpha_{2}(v)\right)\right)=\varphi_{T_{1}}^{\alpha_{1}}(g) v+\varphi_{T_{2}}^{\alpha_{2}}(g) v .
\end{aligned}
$$

This completes the proof of (i).

(ii) Suppose that $\psi \in \operatorname{Hom}_{K}\left(V_{\tau_{2}}, X\right) \otimes \operatorname{Hom}_{K}\left(X, V_{\tau_{1}}\right)$ is a non-zero element which is in the kernel of $\Phi_{X}$. Write $\psi=\sum_{i=1}^{n} \alpha_{i} \otimes T_{i}$ with $n \geq 1$ as small as possible. Then the $\alpha_{i}$ are linearly independent, and also the $T_{i}$ are linearly independent. Now

$$
\sum_{i=1}^{n} \varphi_{T_{i}}^{\alpha_{i}}(g) v=\sum_{i=1}^{n} T_{i}\left(\pi(g)\left(\alpha_{i}(v)\right)\right)=0, \quad \forall g \in G, v \in V_{\tau_{2}}
$$


Writing $g g_{1}$ in the above relation instead of $g$, multiplying the relation by $f\left(g_{1}\right)$ and integrating over $G$ the expression that we obtain in this way, we get

$$
\sum_{i=1}^{n} T_{i}\left(\pi(g) \pi(f)\left(\alpha_{i}(v)\right)\right)=0, \quad \text { for all } \quad g \in G, f \in \mathcal{H}(G), v \in V_{\tau_{2}} .
$$

Suppose now that $\tau_{2}$ is irreducible. Observe that if $v \neq 0$, then the irreducibility of $\tau_{2}$ implies that all $\alpha_{i}(v) \neq 0$. Denote by $l$ the length of the representation $\sum_{i=1}^{n} \operatorname{Im}\left(\alpha_{i}\right)$. Since $\tau_{2}$ is irreducible,

$$
l \leq n .
$$

From the other side, Schur's lemma implies

$$
\operatorname{dim}_{\mathbb{C}} \operatorname{Hom}_{K}\left(V_{\tau_{2}}, \sum_{i=1}^{l} \operatorname{Im}\left(\alpha_{i}\right)\right) \leq l .
$$

We can view $\alpha_{1}, \alpha_{2}, \ldots, \alpha_{n}$ as elements of $\operatorname{Hom}_{K}\left(V_{\tau_{2}}, \sum_{i=1}^{l} \operatorname{Im}\left(\alpha_{i}\right)\right)$. Since they are linearly independent,

$$
n \leq \operatorname{dim}_{\mathbb{C}} \operatorname{Hom}\left(V_{\tau_{2}}, \sum_{i=1}^{l} \operatorname{Im}\left(\alpha_{i}\right)\right) .
$$

Now these three inequalities imply $l=n$. Thus $\sum_{i=1}^{n} \operatorname{Im}\left(\alpha_{i}\right)=\bigoplus_{i=1}^{n} \operatorname{Im}\left(\alpha_{i}\right)$. Now this together with the fact that $\alpha_{i}(v) \in \operatorname{Im}\left(\alpha_{i}\right)$ and $\alpha_{i}(v) \neq 0$ for $v \neq 0$, imply that $\alpha_{1}(v), \alpha_{2}(v), \ldots, \alpha_{n}(v)$ are linearly independent.

Fix $v \neq 0$. Choose $f$ such that $\pi(f)\left(\alpha_{1}(v)\right) \neq 0$ and $\pi(f)\left(\alpha_{i}(v)\right)=0$ for $i>1$. Then $T_{1}\left(\pi(g) \pi(f)\left(\alpha_{1}(v)\right)\right)=0$ for all $g \in G$. The irreducibility of $\pi$ implies $T_{1}=0$. This contradiction completes the proof of (ii) in the lemma if $\tau_{2}$ is irreducible.

We shall now consider $\tau_{2}$ which is not irreducible. Then we can write

$$
\tau_{2}=\tau_{2}^{(1)} \oplus \tau_{2}^{(2)}
$$

where both $\tau_{2}^{(i)}$ are non-trivial. Now obviously

$$
\begin{aligned}
V=\operatorname{Hom}_{\mathbb{C}}\left(V_{\tau_{2}}, V_{\tau_{1}}\right)= & \operatorname{Hom}_{\mathbb{C}}\left(V_{\tau_{2}^{(1)}} \oplus V_{\tau_{2}^{(2)}}, V_{\tau_{1}}\right) \\
& \cong \operatorname{Hom}_{\mathbb{C}}\left(V_{\tau_{2}^{(1)}}, V_{\tau_{1}}\right) \oplus \operatorname{Hom}_{\mathbb{C}}\left(V_{\tau_{2}^{(2)}}, V_{\tau_{1}}\right)=V^{(1)} \oplus V^{(2)},
\end{aligned}
$$

where $V^{(1)}=\operatorname{Hom}_{\mathbb{C}}\left(V_{\tau_{2}^{(1)}}, V_{\tau_{1}}\right)$ and $V^{(2)}=\operatorname{Hom}_{\mathbb{C}}\left(V_{\tau_{2}^{(2)}}, V_{\tau_{1}}\right)$. Let $p^{(i)}: V_{\tau_{2}} \rightarrow V_{\tau_{2}}^{(i)}$ be the corresponding projections (they are $K$-intertwinings). We identify $V^{(1)}$ and $V^{(2)}$ with subspaces of $V$ in a natural way, by identifications $\iota^{(i)}: V^{(i)} \rightarrow V$, $x \mapsto x \circ p^{(i)}$, which obviously satisfy

$$
\iota^{(i)}\left(\tau_{1}\left(k_{1}\right) x \tau_{2}^{(i)}\left(k_{2}\right)\right)=\tau_{1}\left(k_{1}\right) \iota^{(i)}(x) \tau_{2}\left(k_{2}\right)
$$

for $k_{1}, k_{2} \in K, x \in V^{(i)}$.

Denote $\tau^{(1)}=\left(\tau_{1}, \tau_{2}^{(1)}\right)$ and $\tau^{(2)}=\left(\tau_{1}, \tau_{2}^{(2)}\right)$. Composition mapping $f \mapsto \iota^{(i)} \circ f$ (note $\iota^{(i)} \circ f: g \mapsto f(g) \circ p^{(i)}$ ) enables us also to identify $\mathcal{E}_{\omega}\left(G, \tau^{(i)}\right)$ with a subspace of $\mathcal{E}_{\omega}(G, \tau)$ (observe that composing by $\iota^{(i)}$ is a $G$-intertwining, therefore it will preserve infinitesimal character, and further since $\iota^{(i)}$ has the above transformation property, the composing with $\iota^{(i)}$ carries the transformation property with respect to $\tau^{(i)}$ into the transformation property with respect to $\tau$; this explains why 
$\mathcal{E}_{\omega}\left(G, \tau^{(i)}\right)$ goes into $\left.\mathcal{E}_{\omega}(G, \tau)\right)$. Observe that $\mathcal{E}_{\omega}\left(G, \tau^{(1)}\right) \cap \mathcal{E}_{\omega}\left(G, \tau^{(2)}\right)=\{0\}$ since $f \in \mathcal{E}_{\omega}\left(G, \tau^{(1)}\right) \cap \mathcal{E}_{\omega}\left(G, \tau^{(2)}\right)$ takes values in $V^{(1)} \cap V^{(2)}=\{0\}$.

Let $\Phi_{X}^{(i)}$ be the mapping defined by the formula (6-1) when one takes $\tau^{(i)}$ instead of $\tau \cdot \operatorname{Im} \Phi^{(1)} \cap \operatorname{Im} \Phi^{(2)}=\{0\}$ follows from the fact that $\mathcal{E}_{\omega}\left(G, \tau^{(1)}\right) \cap \mathcal{E}_{\omega}\left(G, \tau^{(2)}\right)=$ $\{0\}$. Thus

From the other side

$$
\operatorname{Im} \Phi_{X}^{(1)}+\operatorname{Im} \Phi_{X}^{(2)}=\operatorname{Im} \Phi_{X}^{(1)} \oplus \operatorname{Im} \Phi_{X}^{(2)}
$$

$$
\begin{aligned}
& \operatorname{Hom}_{K}\left(V_{\tau_{2}}, X\right) \otimes \operatorname{Hom}_{K}\left(X, V_{\tau_{1}}\right)=\operatorname{Hom}_{K}\left(V_{\tau_{2}}^{(1)} \oplus V_{\tau_{2}}^{(2)}, X\right) \otimes \operatorname{Hom}_{K}\left(X, V_{\tau_{1}}\right) \\
& =\left(\operatorname{Hom}_{K}\left(V_{\tau_{2}}^{(1)}, X\right) \otimes \operatorname{Hom}_{K}\left(X, V_{\tau_{1}}\right)\right) \oplus\left(\operatorname{Hom}_{K}\left(V_{\tau_{2}}^{(2)}, X\right) \otimes \operatorname{Hom}_{K}\left(X, V_{\tau_{1}}\right)\right)
\end{aligned}
$$

(here we have identified $\operatorname{Hom}_{K}\left(V_{\tau_{2}}^{(i)}, X\right)$ with the subspace of $\operatorname{Hom}_{K}\left(V_{\tau_{2}}, X\right)$ using $\left.\alpha \mapsto \alpha \circ p^{(i)}\right)$. This implies $\operatorname{Im} \Phi_{X}=\operatorname{Im} \Phi_{X}^{(1)}+\operatorname{Im} \Phi_{X}^{(2)}$. Thus

$$
\operatorname{Im} \Phi_{X}=\operatorname{Im} \Phi_{X}^{(1)} \oplus \operatorname{Im} \Phi_{X}^{(2)}
$$

Now we can prove (ii) by induction with respect to the length of $\tau_{2}$. If $\tau_{2}$ is of length one (i.e. if $\tau_{2}$ is irreducible), then we have seen that (ii) holds. If $\tau_{2}$ is not irreducible, we can decompose it into a direct sum of two representations of smaller lengths, and then apply the above relation for $\operatorname{Im} \Phi_{X}$ to conclude injectivity from the inductive assumption.

(iii) Let $\zeta: X \rightarrow X^{\prime}$ be an isomorphism of representations of $G$. Observe that $\alpha \mapsto \zeta \circ \alpha$ is a vector space isomorphism $\operatorname{Hom}_{K}\left(V_{\tau_{2}}, X\right) \rightarrow \operatorname{Hom}_{K}\left(V_{\tau_{2}}, X^{\prime}\right)$, and further $T \mapsto T \circ \zeta^{-1}$ is also a vector space isomorphism $\operatorname{Hom}_{K}\left(X, V_{\tau_{1}}\right) \rightarrow$ $\operatorname{Hom}_{K}\left(X^{\prime}, V_{\tau_{1}}\right)$. Therefore

$$
\alpha \otimes T \mapsto \zeta \circ \alpha \otimes T \circ \zeta^{-1}
$$

extends to an isomorphism

$$
\operatorname{Hom}_{K}\left(V_{\tau_{2}}, X\right) \otimes \operatorname{Hom}_{K}\left(X, V_{\tau_{1}}\right) \rightarrow \operatorname{Hom}_{K}\left(V_{\tau_{2}}, X^{\prime}\right) \otimes \operatorname{Hom}_{K}\left(X^{\prime}, V_{\tau_{1}}\right) .
$$

Recall that

$$
\left(\Phi_{X}(\alpha \otimes T)\right)(g) v=\varphi_{T}^{\alpha}(g) v=T(\pi(g)(\alpha(v))) .
$$

From the other side we have

$$
\begin{gathered}
\left(\Phi_{X^{\prime}}\left(\zeta \circ \alpha \otimes T \circ \zeta^{-1}\right)\right)(g) v=\varphi_{T \circ \zeta^{-1}}^{\zeta \circ \alpha}(g) v=\left(T \circ \zeta^{-1}\right)\left(\pi^{\prime}(g)(\zeta \circ \alpha(v))\right) \\
=\left(T \circ \zeta^{-1}\right)(\zeta \pi(g)(\alpha(v)))=T(\pi(g)(\alpha(v))) .
\end{gathered}
$$

Thus

$$
\Phi_{X}(\alpha \otimes T)=\Phi_{X^{\prime}}\left(\zeta \circ \alpha \otimes T \circ \zeta^{-1}\right)
$$

which implies $\operatorname{Im} \Phi_{X}=\operatorname{Im} \Phi_{X^{\prime}}$. The proof is now complete

Let $\omega$ be the infinitesimal character corresponding to $(M, \rho) \in \Omega(G)$, where $M$ is the standard Levi subgroup of a standard parabolic subgroup $P$. In the following theorem we shall consider mapping (6-1) in the case when $X$ is the induced representation $\operatorname{Hom}_{K}\left(\tau_{i}, \operatorname{Ind}_{P}^{G}(\tilde{\rho})\right)$. This is the reason that we shall need to better understand $\operatorname{Hom}_{K}\left(\tau_{i}, \operatorname{Ind}_{P}^{G}(\tilde{\rho})\right)$.

First Frobenius reciprocity implies

$$
\operatorname{Hom}_{K}\left(\tau_{i}, \operatorname{Ind}_{P}^{G}(\tilde{\rho})\right) \cong \operatorname{Hom}_{K}\left(\tau_{i}, \operatorname{Ind}_{P \cap K}^{K}(\tilde{\rho})\right) \cong \operatorname{Hom}_{P \cap K}\left(\tau_{i}, \tilde{\rho}\right)
$$

(the first isomorphism is given simply by restriction of functions from $\operatorname{Ind}_{P}^{G}(\tilde{\rho})$ to $K$, while the second one is Frobenius reciprocity). 
Observe that $\tau_{i} \mid(P \cap K)=\tau_{i}^{N \cap K} \oplus \tau_{i, N \cap K}$, where $\tau_{i}^{N \cap K}$ denotes $N \cap K$ invariants and $\tau_{i, N \cap K}$ denotes $N \cap K$-coinvariants of $\tau_{i}$. These two spaces are $P \cap K$-subrepresentations since $P \cap K$ normalizes $N \cap K$. Denote by

$$
p_{i}^{N \cap K}
$$

the projection of $V_{\tau_{i}}$ onto $\tau_{i}^{N \cap K}$ along $\tau_{i, N \cap K}$ (it is given by a well-known integral formula $p_{i}^{N \cap K}=\int_{N \cap K} \tau_{i}(n) d n$, where the Haar measure on $N \cap K$ is normalized). Clearly, $p_{i}^{N \cap K}$ are $P \cap K$-intertwinings.

Now since the action of $\tilde{\rho}$ in (6-2) is defined to be trivial on $N$, each element in $\operatorname{Hom}_{P \cap K}\left(\tau_{i}, \tilde{\rho}\right)$ vanishes on $N \cap K$-coinvariants, and therefore we get a natural mapping from $\operatorname{Hom}_{P \cap K}\left(\tau_{i}, \tilde{\rho}\right)$ into $\operatorname{Hom}_{P \cap K}\left(\tau_{i}^{N \cap K}, \tilde{\rho}\right)$, which one easily sees that it is an isomorphism.

Observe that $\operatorname{Hom}_{P \cap K}\left(\tau_{i}^{N \cap K}, \tilde{\rho}\right) \subseteq \operatorname{Hom}_{M \cap K}\left(\tau_{i}^{N \cap K}, \tilde{\rho}\right)$. Since $(M \cap K)(P \cap K)=$ $P \cap K$ (see (4-2)), we have here the equality.

Therefore we have seen that

$$
\operatorname{Hom}_{P \cap K}\left(\tau_{i}, \tilde{\rho}\right) \cong \operatorname{Hom}_{P \cap K}\left(\tau_{i}^{N \cap K}, \tilde{\rho}\right)=\operatorname{Hom}_{M \cap K}\left(\tau_{i}^{N \cap K}, \tilde{\rho}\right) .
$$

From (6-2) and (6-3) we get

$$
\operatorname{Hom}_{K}\left(\tau_{i}, \operatorname{Ind}_{P}^{G}(\tilde{\rho})\right) \cong \operatorname{Hom}_{M \cap K}\left(\tau_{i}^{N \cap K}, \tilde{\rho}\right) .
$$

Now we can prove the following:

6.4. Theorem. If $\omega$ is an irreducible and semi-simple infinitesimal character of $G$ corresponding to the evaluation in $(M, \rho) \in \Omega(G)$, then

$$
\operatorname{dim}_{\mathbb{C}}\left(\mathcal{E}_{\omega}(G, \tau)\right)=\operatorname{dim}_{\mathbb{C}}\left(\operatorname{Hom}_{M \cap K}\left(\tau_{1}^{N \cap K}, \tilde{\rho}\right)\right) \operatorname{dim}_{\mathbb{C}}\left(\operatorname{Hom}_{M \cap K}\left(\tau_{2}^{N \cap K}, \tilde{\rho}\right)\right) .
$$

Proof. Observe first that Proposition 5.4 and (iv) of Proposition 6.1 imply that that there exists an isomorphism

$$
\lambda: \operatorname{Hom}_{\mathcal{H}(K)}\left(\left(\mathcal{H}(G) \otimes_{\mathcal{H}(K)} V_{\tau_{2}}\right)^{\langle\tilde{\omega}\rangle}, V_{\tau_{1}}\right) \rightarrow \mathcal{E}_{\omega}(G, \tau)
$$

Suppose

$$
\operatorname{Hom}_{K}\left(V_{\tau_{2}}, \operatorname{Ind}_{P}^{G}(\tilde{\rho})\right)=\{0\} .
$$

Then (v) of Remarks 6.2 implies $\left(\mathcal{H}(G) \otimes_{\mathcal{H}(K)} V_{\tau_{2}}\right)^{\langle\tilde{\omega}\rangle}=\{0\}$. Now the fact that $\lambda$ (from $(6-5))$ is an isomorphism implies $\operatorname{dim}_{\mathbb{C}}\left(\mathcal{E}_{\omega}(G, \tau)\right)=\{0\}$. From the other side, (6-6) and (6-4) imply $\operatorname{dim}_{\mathbb{C}}\left(\operatorname{Hom}_{M \cap K}\left(\tau_{2}^{N \cap K}, \tilde{\rho}\right)\right)=0$. Therefore, the claim of the theorem holds in this case.

It remains to consider the case

$$
\operatorname{Hom}_{K}\left(V_{\tau_{2}}, \operatorname{Ind}_{P}^{G}(\tilde{\rho})\right) \neq\{0\} .
$$

We shall assume this in the sequel. Now (v) of Remarks 6.2 implies

$$
\left(\mathcal{H}(G) \otimes_{\mathcal{H}(K)} V_{\tau_{2}}\right)^{\langle\tilde{\omega}\rangle} \neq\{0\} .
$$

Recall that $\bar{e}_{K_{0}} \otimes \in \operatorname{Hom}_{\mathcal{H}(K)}\left(V_{\tau_{2}},\left(\mathcal{H}(G) \otimes_{\mathcal{H}(K)} V_{\tau_{2}}\right)^{\langle\tilde{\omega}\rangle}\right)$. Note that by (iii) of Remarks 6.2 , the isomorphism $\lambda$ is just $\Phi_{\left(\mathcal{H}(G) \otimes_{\mathcal{H}(K)} V_{\tau_{2}}\right)\langle\tilde{\omega}\rangle}$ restricted to

$$
\left\{\bar{e}_{K_{0}} \otimes\right\} \times \operatorname{Hom}_{\mathcal{H}(K)}\left(\left(\mathcal{H}(G) \otimes_{\mathcal{H}(K)} V_{\tau_{2}}\right)^{\langle\tilde{\omega}\rangle}, V_{\tau_{1}}\right) .
$$

Therefore $\Phi_{\left(\mathcal{H}(G) \otimes_{\mathcal{H}(K)} V_{\tau_{2}}\right)\langle\tilde{\omega}\rangle}$ must be an epimorphism.

Recall that $\left(\mathcal{H}(G) \otimes_{\mathcal{H}(K)} V_{\tau_{2}}\right)^{\langle\tilde{\omega}\rangle}$ is by Lemma 5.1 a finitely generated smooth representation, and it has infinitesimal character (which is $\tilde{\omega}$ ). Theorem 3.6 implies 
that it is admissible. Since infinitesimal character is $\tilde{\omega}$, each irreducible subquotient of the representation $\left(\mathcal{H}(G) \otimes_{\mathcal{H}(K)} V_{\tau_{2}}\right)^{\langle\tilde{\omega}\rangle}$ is isomorphic to an irreducible subquotient of $\operatorname{Ind}_{P}^{G}(\tilde{\rho})$. The last representation is irreducible by the assumptions of the theorem. Therefore each irreducible subquotient of $\left(\mathcal{H}(G) \otimes_{\mathcal{H}(K)} V_{\tau_{2}}\right)^{\langle\tilde{\omega}\rangle}$ is isomorphic to $\operatorname{Ind}_{P}^{G}(\tilde{\rho})$. Since $\tilde{\omega}$ is semi-simple infinitesimal character (because $\omega$ is semisimple), $\left(\mathcal{H}(G) \otimes_{\mathcal{H}(K)} V_{\tau_{2}}\right)^{\langle\tilde{\omega}\rangle}$ is isomorphic to a direct sum of finitely many (irreducible) representations, each of which is isomorphic to $\operatorname{Ind}_{P}^{G}(\tilde{\rho})$. Note that this sum contains at least one irreducible representation since $\left(\mathcal{H}(G) \otimes_{\mathcal{H}(K)} V_{\tau_{2}}\right)^{\langle\tilde{\omega}\rangle} \neq\{0\}$.

Now (i) and (iii) of Lemma 6.3 imply that $\Phi_{\operatorname{Ind}_{P}^{G}(\tilde{\rho})}$ is a surjective linear mapping

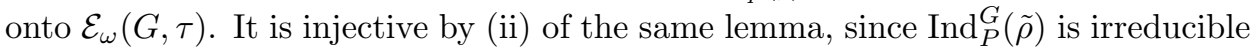
by the assumption. Therefore, $\Phi_{\operatorname{Ind}_{P}^{G}(\tilde{\rho})}$ is an isomorphism of

$$
\operatorname{Hom}_{K}\left(V_{\tau_{2}}, \operatorname{Ind}_{P}^{G}(\tilde{\rho})\right) \otimes \operatorname{Hom}_{K}\left(\operatorname{Ind}_{P}^{G}(\tilde{\rho}), V_{\tau_{1}}\right)
$$

onto $\mathcal{E}_{\omega}(G, \tau)$.

One directly sees that

$$
\operatorname{dim}_{\mathbb{C}} \operatorname{Hom}_{K}\left(\operatorname{Ind}_{P}^{G}(\tilde{\rho}), V_{\tau_{1}}\right)=\operatorname{dim}_{\mathbb{C}} \operatorname{Hom}_{K}\left(V_{\tau_{1}}, \operatorname{Ind}_{P}^{G}(\tilde{\rho})\right) .
$$

Now this and (6-4) imply the claim of the theorem. The proof is now complete.

6.5. Remarks. (i) Theorem 3.9 states that the conditions required in the above theorem hold on a Zariski open dense subset of infinitesimal characters $\Omega(G)$.

(ii) The formula in the theorem and Theorem 3.9 imply that the dimension of the space of generalized functions $\mathcal{E}_{\omega}(G, \tau)$ is constant on a Zariski open dense subset of each connected component of $\Omega(G)$.

\section{EisEnstein INTEGRALS}

In the sixth section, as a consequence of the study of isomorphisms related to the induced representations and intertwinings, we got Theorem 6.4. The most important among these isomorphisms are Frobenius isomorphisms. Moreover, we can explicitly write these isomorphism, and get explicit formulas for the generalized spherical functions. By these explicit formulas, generalized spherical functions are given by integrals. The integrals in these formulas for generalized spherical functions come from the isomorphism $\operatorname{Ind}_{P}^{G}(\rho)^{\sim} \cong \operatorname{Ind}_{P}^{G}(\tilde{\rho})$, which is given by integrating over $K$. Since these integrals are Eisenstein integrals, in this way one gets generalized spherical functions as Eisenstein integrals (on a big set of infinitesimal characters). So, in this way Eisenstein integrals naturally arise in this setting (and background of them are Frobenius isomorphisms and the formula for isomorphism $\left.\operatorname{Ind}_{P}^{G}(\rho)^{\sim} \cong \operatorname{Ind}_{P}^{G}(\tilde{\rho})\right)$.

In this section we shall carefully trace the isomorphisms that we mention above, and at the end get the Eisenstein integral formulas.

Let $P=M N$ be a standard parabolic subgroup with standard Levi decomposition. If we decompose $g \in G$ in a product

$$
g=m n k, \quad m \in M, n \in N, k \in K,
$$

then we shall write

$$
\mathbf{m}_{r}(g)=m, \mathbf{n}_{r}(g)=n, \mathbf{k}_{r}(g)=k .
$$

Note that $\mathbf{m}_{r}(g), \mathbf{n}_{r}(g), \mathbf{k}_{r}(g)$ are not uniquely determined by $g$. 
We shall denote by

$$
\kappa_{V_{\tau_{1}}}: V_{\tau_{1}} \rightarrow \widetilde{\widetilde{V}}_{\tau_{1}}
$$

the canonical isomorphism

$$
\left[\kappa_{V_{\tau_{1}}}\left(v_{1}\right)\right]\left(\tilde{v}_{1}\right)=\tilde{v}_{1}\left(v_{1}\right),
$$

which is an equivalence of representations of $K$.

Let $(\pi, X)$ be an admissible representation of $G$. In the same way we denote by $\kappa_{X}: X \rightarrow \widetilde{\widetilde{X}}$ the canonical isomorphism $\left[\kappa_{X}(y)\right](\tilde{y})=\tilde{y}(y)$ of representations of $G$.

We define

$$
S \mapsto \bar{S}, \quad \operatorname{Hom}_{K}\left(\widetilde{V}_{\tau_{1}}, \widetilde{X}\right) \rightarrow \operatorname{Hom}_{K}\left(X, V_{\tau_{1}}\right)
$$

by

$$
\bar{S}(y)=\kappa_{V_{\tau_{1}}}^{-1}\left(\kappa_{X}(y) \circ S\right) .
$$

Now we shall show that $S \mapsto \bar{S}$ carries $\operatorname{Hom}_{K}\left(\widetilde{V}_{\tau_{1}}, \widetilde{X}\right)$ into $\operatorname{Hom}_{K}\left(X, V_{\tau_{1}}\right)$ :

$$
\begin{aligned}
\bar{S}(\pi(k) y)= & \kappa_{V_{\tau_{1}}}^{-1}\left(\kappa_{X}(\pi(k) y) \circ S\right)=\kappa_{V_{\tau_{1}}}^{-1}\left(\tilde{\tilde{\pi}}(k)\left(\kappa_{X}(y)\right) \circ S\right) \\
= & \kappa_{V_{\tau_{1}}}^{-1}\left(\kappa_{X}(y) \circ \tilde{\pi}\left(k^{-1}\right) \circ S\right)=\kappa_{V_{\tau_{1}}}^{-1}\left(\kappa_{X}(y) \circ S \circ \tilde{\tau}_{1}\left(k^{-1}\right)\right) \\
& =\kappa_{V_{\tau_{1}}}^{-1}\left(\tilde{\tau}_{1}(k)\left(\kappa_{X}(y) \circ S\right)\right)=\tau_{1}(k) \kappa_{V_{\tau_{1}}}^{-1}\left(\kappa_{X}(y) \circ S\right)=\tau_{1}(k) \bar{S}(y) .
\end{aligned}
$$

We shall show that $S \mapsto \bar{S}$ is injective. Suppose $\bar{S}=0$. Then for every $y \in X$ we have $0=\bar{S}(y)=\kappa_{V_{\tau_{1}}}^{-1}\left(\kappa_{X}(y) \circ S\right)$, which implies $\kappa_{X}(y) \circ S=0$ and further $S=0$. Since $\operatorname{Hom}_{K}\left(\widetilde{V}_{\tau_{1}}, \widetilde{X}\right)$ and $\operatorname{Hom}_{K}\left(X, V_{\tau_{1}}\right)$ have the same (finite) dimension, we get that (7-1) is an isomorphism.

Suppose now that $(\pi, X)$ has an infinitesimal character. Now for

$$
\alpha \in \operatorname{Hom}_{K}\left(V_{\tau_{2}}, X\right), \quad S \in \operatorname{Hom}_{K}\left(\widetilde{V}_{\tau_{1}}, \widetilde{X}\right)
$$

we have by the definition of the previous section

$$
\Phi \frac{\alpha}{S}(g) v_{2}=\bar{S}\left(\pi(g)\left(\alpha\left(v_{2}\right)\right)\right)=\kappa_{\tau_{\tau_{1}}}^{-1}\left[\kappa_{X}\left(\pi(g)\left(\alpha\left(v_{2}\right)\right)\right) \circ S\right] .
$$

If the infinitesimal character of $\pi$ is $\omega$, then the above generalized spherical function corresponds to the infinitesimal character $\tilde{\omega}$.

The above formula implies

$$
\kappa_{V_{\tau_{1}}}\left(\Phi \frac{\alpha}{S}(g) v_{2}\right)=\bar{S}\left(\pi(g)\left(\alpha\left(v_{2}\right)\right)\right)=\kappa_{X}\left[\pi(g)\left(\alpha\left(v_{2}\right)\right)\right] \circ S
$$

and further

$$
\left[\kappa_{V_{\tau_{1}}}\left(\Phi \frac{\alpha}{S}(g) v_{2}\right)\right]\left(\tilde{v}_{1}\right)=\kappa_{X}\left[\pi(g)\left(\alpha\left(v_{2}\right)\right)\right] \circ S\left(\tilde{v}_{1}\right)=S\left(\tilde{v}_{1}\right)\left[\pi(g)\left(\alpha\left(v_{2}\right)\right)\right] .
$$

Now we shall assume that $(\sigma, Y)$ is an admissible representation of $M$ with infinitesimal character $\omega_{\sigma}$. Let $\omega_{\sigma}$ correspond to the evaluation at $\left(M_{\sigma}, \rho_{\sigma}\right) \in$ $\Omega(M)$. Then Proposition 3.8 implies that $\operatorname{Ind}_{P}^{G}(\sigma)$ has the infinitesimal character which corresponds to the evaluation in $\left(M_{\sigma}, \rho_{\sigma}\right) \in \Omega(G)$.

We shall assume below that $X=\operatorname{Ind}_{P}^{G}(\sigma)$. Consider isomorphisms (6-2) and (6-3):

$$
\begin{aligned}
\operatorname{Hom}_{M \cap K}\left(\tau_{2}^{N \cap K}, \sigma\right) & \cong \operatorname{Hom}_{P \cap K}\left(\tau_{2}, \sigma\right) \cong \operatorname{Hom}_{K}\left(\tau_{2}, \operatorname{Ind}_{P \cap K}^{K}(\sigma)\right) \\
& \cong \operatorname{Hom}_{K}\left(\tau_{2}, \operatorname{Ind}_{P}^{G}(\sigma)\right) .
\end{aligned}
$$


Denote the resulting isomorphism by

$$
\beta \mapsto \beta^{\prime} .
$$

Note that the first two isomorphisms above are given by

$$
\beta \mapsto \beta \circ p_{2}^{N \cap K} \mapsto\left[v_{2} \mapsto \beta \circ p_{2}^{N \cap K}\left(\tau_{2}(k) v_{2}\right)\right] .
$$

Thus

$$
\left[\beta^{\prime}\left(v_{2}\right)\right](g)=\delta_{P}^{1 / 2}\left(\mathbf{m}_{r}(g)\right) \sigma\left(\mathbf{m}_{r}(g)\right) \beta \circ p_{2}^{N \cap K}\left(\tau_{2}\left(\mathbf{k}_{r}(g)\right) v_{2}\right),
$$

where $\delta_{P}$ denotes the modular function of $P$.

We shall also need the isomorphism

$$
\operatorname{Hom}_{M \cap K}\left(\tilde{\tau}_{1}^{N \cap K}, \tilde{\sigma}\right) \cong \operatorname{Hom}_{K}\left(\tilde{\tau}_{1}, \operatorname{Ind}_{P}^{G}(\tilde{\sigma})\right),
$$

which will be denoted by $U \mapsto U^{\prime}$. Thus

$$
\left[U^{\prime}\left(\tilde{v}_{1}\right)\right](g)=\delta_{P}^{1 / 2}\left(\mathbf{m}_{r}(g)\right) \tilde{\sigma}\left(\mathbf{m}_{r}(g)\right) U \circ \tilde{p}_{1}^{N \cap K}\left(\tilde{\tau}_{1}\left(\mathbf{k}_{r}(g)\right) \tilde{v}_{1}\right)
$$

(in the above formula, $\tilde{p}_{1}^{N \cap K}$ is the natural projection of $\widetilde{V}_{\tau_{1}}$ onto $N \cap K$-invariants). The composition of $U \mapsto U^{\prime}$ with the isomorphism

$$
\operatorname{Hom}_{K}\left(\tilde{\tau}_{1}, \operatorname{Ind}_{P}^{G}(\tilde{\sigma})\right) \cong \operatorname{Hom}_{K}\left(\tilde{\tau}_{1}, \operatorname{Ind}_{P}^{G}(\sigma)^{\Upsilon}\right)
$$

will be denoted by $U \mapsto U^{\prime \prime}$. Now for $f \in \operatorname{Ind}_{P}^{G}(\sigma)$ we have

$$
\left[U^{\prime \prime}\left(\tilde{v}_{1}\right)\right](f)=\int_{K}\left[\left(U^{\prime}\left(\tilde{v}_{1}\right)\right)(k)\right](f(k)) d k .
$$

Let $\beta \in \operatorname{Hom}_{M \cap K}\left(\tau_{2}^{N \cap K}, \sigma\right)$ and $U \in \operatorname{Hom}_{M \cap K}\left(\tilde{\tau}_{1}^{N \cap K}, \tilde{\sigma}\right)$. Then by $(7-2)$

$$
\begin{gathered}
{\left[\kappa_{V_{\tau_{1}}}\left(\Phi_{U^{\prime \prime}}^{\beta^{\prime}}(g) v_{2}\right)\right]\left(\tilde{v}_{1}\right)=U^{\prime \prime}\left(\tilde{v}_{1}\right)\left[R_{g}\left(\beta^{\prime}\left(v_{2}\right)\right)\right]} \\
=\int_{K}\left[\left(U^{\prime}\left(\tilde{v}_{1}\right)\right)(k)\right]\left(R_{g}\left(\beta^{\prime}\left(v_{2}\right)\right)(k)\right) d k \\
=\int_{K}\left[\left(U^{\prime}\left(\tilde{v}_{1}\right)\right)(k)\right]\left(\left(\beta^{\prime}\left(v_{2}\right)\right)(k g)\right) d k \\
=\int_{K}\left[U \circ \tilde{p}_{1}^{N \cap K}\left(\tilde{\tau}_{1}(k) \tilde{v}_{1}\right)\right]\left(\left(\beta^{\prime}\left(v_{2}\right)\right)(k g)\right) d k \\
=\int_{K}\left[U \circ \tilde{p}_{1}^{N \cap K}\left(\tilde{\tau}_{1}(k) \tilde{v}_{1}\right)\right]\left(\delta_{P}^{1 / 2}\left(\mathbf{m}_{r}(k g)\right) \sigma\left(\mathbf{m}_{r}(k g)\right) \beta \circ p_{2}^{N \cap K}\left(\tau_{2}\left(\mathbf{k}_{r}(k g)\right) v_{2}\right)\right) d k .
\end{gathered}
$$

Recall that (7-2) implies for $m \in M$ :

$$
\left[\kappa_{V_{\tau_{1}}^{N \cap K}}\left(\Phi_{\bar{U}}^{\beta}(m) p_{2}^{N \cap K} v_{2}\right)\right]\left(\tilde{p}_{1}^{N \cap K} \tilde{v}_{1}\right)=U\left(\tilde{p}_{1}^{N \cap K} \tilde{v}_{1}\right)\left[\sigma(m)\left(\beta\left(p_{2}^{N \cap K} v_{2}\right)\right)\right] .
$$

Thus

$$
\begin{aligned}
& {\left[\kappa_{V_{\tau_{1}}}\left(\Phi_{\bar{U}^{\prime \prime}}^{\beta^{\prime}}(g) v_{2}\right)\right]\left(\tilde{v}_{1}\right) } \\
= & \int_{K} \delta_{P}^{1 / 2}\left(\mathbf{m}_{r}(k g)\right)\left[\kappa_{V_{\tau_{1}}^{N \cap K}}\left(\Phi_{\bar{U}}^{\beta}\left(\mathbf{m}_{r}(k g)\right) p_{2}^{N \cap K} \tau_{2}\left(\mathbf{k}_{r}(k g)\right) v_{2}\right)\right]\left(\tilde{p}_{1}^{N \cap K} \tilde{\tau}_{1}(k) \tilde{v}_{1}\right) d k,
\end{aligned}
$$


and further

$$
\begin{aligned}
\tilde{v}_{1} & \left(\Phi_{\overline{U^{\prime \prime}}}^{\beta^{\prime}}(g) v_{2}\right) \\
& =\int_{K} \delta_{P}^{1 / 2}\left(\mathbf{m}_{r}(k g)\right)\left(\tilde{p}_{1}^{N \cap K} \tilde{\tau}_{1}(k) \tilde{v}_{1}\right)\left(\Phi_{\bar{U}}^{\beta}\left(\mathbf{m}_{r}(k g)\right) p_{2}^{N \cap K} \tau_{2}\left(\mathbf{k}_{r}(k g)\right) v_{2}\right) d k \\
& =\int_{K} \delta_{P}^{1 / 2}\left(\mathbf{m}_{r}(k g)\right)\left(\tilde{\tau}_{1}(k) \tilde{v}_{1}\right)\left(\Phi_{\bar{U}}^{\beta}\left(\mathbf{m}_{r}(k g)\right) p_{2}^{N \cap K} \tau_{2}\left(\mathbf{k}_{r}(k g)\right) v_{2}\right) d k \\
& =\int_{K} \delta_{P}^{1 / 2}\left(\mathbf{m}_{r}(k g)\right) \tilde{v}_{1}\left(\tau_{1}\left(k^{-1}\right) \Phi_{\bar{U}}^{\beta}\left(\mathbf{m}_{r}(k g)\right) p_{2}^{N \cap K} \tau_{2}\left(\mathbf{k}_{r}(k g)\right) v_{2}\right) d k
\end{aligned}
$$

Thus

$$
\Phi_{\overline{U^{\prime \prime}}}^{\beta^{\prime}}(g) v_{2}=\int_{K} \delta_{P}^{1 / 2}\left(\mathbf{m}_{r}(k g)\right) \tau_{1}\left(k^{-1}\right) \Phi_{\bar{U}}^{\beta}\left(\mathbf{m}_{r}(k g)\right) p_{2}^{N \cap K} \tau_{2}\left(\mathbf{k}_{r}(k g)\right) v_{2} d k .
$$

Denote

$$
\tau^{N \cap K}=\left(\tau_{1}^{N \cap K}, \tau_{2}^{N \cap K}\right)
$$

For $\psi \in C\left(M, \tau^{N \cap K}\right)$ define a function $\psi_{r}: G \rightarrow V$ by the formula

$$
\psi_{r}(g)\left(v_{2}\right)=\psi\left(\mathbf{m}_{r}(g)\right) p_{2}^{N \cap K}\left(\tau_{2}\left(\mathbf{k}_{r}(g)\right) v_{2}\right)
$$

and define $\delta_{P, r}: G \rightarrow \mathbb{C}$ by

$$
\delta_{P, r}(g)=\delta_{P}\left(\mathbf{m}_{r}(g)\right)
$$

Let us show that this is well defined. Suppose $m_{1} n_{1} k_{1}=m_{2} n_{2} k_{2}$. Then $k_{2} k_{1}^{-1}=$ $n_{2}^{-1} m_{2}^{-1} m_{1} n_{1}=m_{2}^{-1} m_{1}\left(m_{1}^{-1} m_{2} n_{2}^{-1} m_{2}^{-1} m_{1}\right) n_{1} \in P \cap K$, where $m_{2}^{-1} m_{1} \in M \cap K$ and $\left(m_{1}^{-1} m_{2} n_{2}^{-1} m_{2}^{-1} m_{1}\right) n_{1} \in N \cap K$. This implies that $k_{2} k_{1}^{-1}=n^{\prime} m_{2}^{-1} m_{1}$ for some $n^{\prime} \in N \cap K$. Therefore

$$
\begin{aligned}
& \psi\left(m_{2}\right) p_{2}^{N \cap K}\left(\tau_{2}\left(k_{2}\right) v_{2}\right)=\psi\left(m_{2}\right) p_{2}^{N \cap K}\left(\tau_{2}\left(k_{2} k_{1}^{-1} k_{1}\right) v_{2}\right) \\
= & \psi\left(m_{2}\right) p_{2}^{N \cap K}\left(\tau_{2}\left(n^{\prime}\right) \tau_{2}\left(m_{2}^{-1} m_{1}\right) \tau_{2}\left(k_{1}\right) v_{2}\right)=\psi\left(m_{2}\right) \tau_{2}^{N \cap K}\left(m_{2}^{-1} m_{1}\right) p_{2}^{N \cap K}\left(\tau_{2}\left(k_{1}\right) v_{2}\right) \\
= & \psi\left(m_{2} m_{2}^{-1} m_{1}\right) p_{2}^{N \cap K}\left(\tau_{2}\left(k_{1}\right) v_{2}\right)=\psi\left(m_{1}\right) p_{2}^{N \cap K}\left(\tau_{2}\left(k_{1}\right) v_{2}\right) .
\end{aligned}
$$

This proves that $\psi_{r}$ is well defined.

Denote $\psi=\Phi_{\bar{U}}^{\beta}$. Note $\psi \in \mathcal{E}_{\tilde{\omega}_{\sigma}}\left(M, \tau^{N \cap K}\right)$ and

$$
\Phi_{\overline{U^{\prime \prime}}}^{\beta^{\prime}}(g) v_{2}=\int_{K} \delta_{P, r}^{1 / 2}(k g) \tau_{1}\left(k^{-1}\right) \psi_{r}(k g) v_{2} d k .
$$

Since from each spherical function in $\mathcal{E}_{\omega_{\sigma}}\left(M, \tau^{N \cap K}\right)$ we can get some smooth representation with central character $\omega_{\sigma}$ (see (i) of Proposition 6.1), we have proved the first claim of the following

7.1. Theorem. (i) Let $\omega^{\prime}$ be an infinitesimal character of $M$ determined by $\left(M^{\prime}, \rho^{\prime}\right) \in \Omega(M)$. Let $\omega^{\prime \prime}$ be an infinitesimal character of $G$ determined by $\left(M^{\prime}, \rho^{\prime}\right) \in$ $\Omega(G)$. Define the function $\delta_{P, r}$ on $G$ by

$$
\delta_{P, r}(g)=\delta_{P}\left(\mathbf{m}_{r}(g)\right)
$$

For $\psi \in \mathcal{E}_{\omega^{\prime}}\left(M, \tau^{N \cap K}\right)$ set

$$
\psi_{r}(g)=\psi\left(\mathbf{m}_{r}(g)\right) p_{2}^{N \cap K} \tau_{2}\left(\mathbf{k}_{r}(g)\right) .
$$

Then $\psi_{r}$ is well defined and if we define $E_{P}$ by the formula

$$
E_{P}(\psi, g)=\int_{K} \delta_{P, r}^{1 / 2}(k g) \tau_{1}\left(k^{-1}\right) \psi_{r}(k g) d k,
$$


then $E_{P}$ defines a linear mapping

$$
E_{P}: \mathcal{E}_{\omega^{\prime}}\left(M, \tau^{N \cap K}\right) \rightarrow \mathcal{E}_{\omega^{\prime \prime}}(G, \tau)
$$

(ii) Let $\omega$ be a semi-simple irreducible infinitesimal character of $G$ corresponding to $(M, \rho) \in \Omega(G)$ and let $\omega_{\rho}$ be the infinitesimal character of $M$ corresponding to $(M, \rho) \in \Omega(M)$. Then

$$
E_{P}: \mathcal{E}_{\omega_{\rho}}\left(M, \tau^{N \cap K}\right) \rightarrow \mathcal{E}_{\omega}(G, \tau)
$$

is an isomorphism.

Proof. (ii) We have seen in the sixth section that $E_{P}$ is surjective in this case. The proof of Theorem 6.4 implies (ii).

7.2. Remarks. (i) Note that (ii) of the above theorem reduces the problem of describing generalized spherical functions for semi-simple irreducible infinitesimal characters of $G$ to the generalized spherical functions with cuspidal infinitesimal character.

It would be interesting to find such a reduction when one does not have semisimplicity and irreducibility of the infinitesimal character.

(ii) In this section we could have worked with induced representations, realized in a way that the group acts by left translations in the induced representations. In this way we would get slightly different integral formulas for generalized spherical functions corresponding to non-cuspidal infinitesimal characters, in which $g$ would precede $k$ (while in our formula $g$ comes after $k$ ).

\section{SEMI-SIMPLICITY}

Observe that the principal series representation, where the trivial (and the Steinberg) representations are subquotients, has infinitesimal character but is not semisimple. In this section we shall prove that this is an exceptional situation. Namely, we shall prove that there exists a dense Zariski open subset of $\Omega(G)$ such that the category of all the smooth representations with the infinitesimal character corresponding to an element of this subset is semi-simple.

We shall start with a simple

8.1. Lemma. Fix an infinitesimal character $\omega$ of $G$. Suppose that in the category $\mathcal{C}_{\omega}$ of all the smooth representations of $G$ with the infinitesimal character $\omega$, each admissible representation of length two is semi-simple. Then the whole category is semi-simple (i.e. each representation in it is semi-simple).

Proof. First we shall show that each admissible representation in the category is semi-simple.

Let $\omega$ correspond to the evaluation in $(M, \rho) \in \Omega(G)$ and let $\pi$ be an admissible representation in $\mathcal{C}_{\omega}$. Since each irreducible representation in $\mathcal{C}_{\omega}$ is equivalent to a subquotient of $\operatorname{Ind}_{P}^{G}(\rho)$, we get that $\pi$ has finite length. Now we shall prove the semi-simplicity of $\pi$ by induction with respect to the length of $\pi$.

If $\pi$ has length one or two, there is nothing to prove. Therefore suppose that $\pi$ has length $n \geq 3$, and that admissible representation in $\mathcal{C}_{\omega}$ of strictly smaller length are semi-simple. Choose an irreducible subrepresentation $\pi^{\prime}$ in $\pi$ of length $n-2$. The assumption of the lemma implies that $\pi / \pi^{\prime}=\tau_{1} \oplus \tau_{2}$ is a direct sum of two irreducible representations. Consider the composition $q_{i}: \pi \rightarrow \pi / \pi^{\prime}=\tau_{1} \oplus \tau_{2} \rightarrow \tau_{i}$. Then the lengths of $\operatorname{Ker}\left(q_{i}\right)$ are both $n-1$, and $\operatorname{Ker}\left(q_{1}\right) \neq \operatorname{Ker}\left(q_{2}\right)$. This implies 
$\operatorname{Ker}\left(q_{1}\right)+\operatorname{Ker}\left(q_{2}\right)=\pi$. Now the inductive assumption implies that $\pi$ is generated by irreducible subrepresentations. Thus, it is semi-simple.

Suppose now that $(\pi, V)$ is a smooth representation in $\mathcal{C}_{\omega}$. Let $v \in V$. Denote by $V^{\prime}$ the subrepresentation of $V$ generated by $v$. Then $V^{\prime}$ is admissible by Theorem 3.6. By the first part of the proof, $V^{\prime}$ is a sum of irreducible subrepresentations. Therefore, the whole $V$ is generated by the irreducible subrepresentations. Thus, $(\pi, V)$ is semi-simple. This completes the proof.

Let $\Sigma$ be the set of roots of $A_{\emptyset}$ in $G$ and denote by $\Sigma^{+} \subset \Sigma$ (resp. $\Delta \subset \Sigma^{+}$) the set of positive (resp. simple) roots determined by the choice of the minimal parabolic subgroup $P_{\emptyset}$ (which we have fixed). Recall that we have bijection between subsets of $\Delta$ and standard parabolic subgroups of $G$. For $\Theta \subseteq \Delta$ we shall denote by $P_{\Theta}=M_{\Theta} N_{\Theta}$ the corresponding standard parabolic subgroup. We shall assume that $M_{\Theta}$ is the standard Levi subgroup, i.e. $M_{\emptyset} \subseteq M_{\Theta}$.

Let $W(\Theta):=\{w \in W \mid w(\Theta)=\Theta\}$ and let $W_{\Theta}$ be the subgroup of $W$ generated by the reflections corresponding to the elements of $\Theta$. The subgroup $W(\Theta)$ normalizes $W_{\Theta}$. Set

$$
W^{\Theta}=W(\Theta) / W_{\Theta} .
$$

For a representation $\sigma$ of $M$ and $w \in W^{\Theta}$ denote by $w \sigma$ the representation of $M$ defined by

$$
(w \sigma)(m)=\sigma\left(w^{-1} m w\right) .
$$

Note that $w \sigma$ is determined up to an equivalence.

We shall fix $\Theta \subseteq \Delta$ and denote

$$
P=P_{\Theta} \quad \text { and } \quad M=M_{\theta}
$$

below.

For a smooth representation $\pi$ of $G$, Frobenius reciprocity

$$
\operatorname{Hom}_{M}\left(\mathrm{r}_{M}^{G}(\pi), \mathrm{r}_{M}^{G}(\pi)\right) \cong \operatorname{Hom}_{G}\left(\pi, \operatorname{Ind}_{P}^{G}\left(r_{M}^{G}(\pi)\right)\right)
$$

is a canonical isomorphism. By Frobenius isomorphism, the identity homomorphism of the left-hand side corresponds to a $G$-intertwining of the right hand side, which we shall denote by

$$
\mathfrak{I}_{\pi}: \pi \rightarrow \operatorname{Ind}_{P}^{G}\left(\mathrm{r}_{M}^{G}(\pi)\right) .
$$

Observe that $\mathfrak{I}_{\pi}$ is non-zero if $\mathrm{r}_{M}^{G}(\pi)$ is non-zero. Since $\operatorname{Ind}_{P}^{G}$ and $\mathrm{r}_{M}^{G}(\pi)$ are adjoint functors, $\mathfrak{I}_{\pi}$ defines a natural transform between identity functor and the functor $\operatorname{Ind}_{P}^{G} \circ \mathrm{r}_{M}^{G}$, i.e. if $\varphi: \pi_{1} \rightarrow \pi_{2}$ is a $G$-intertwining, then the diagram

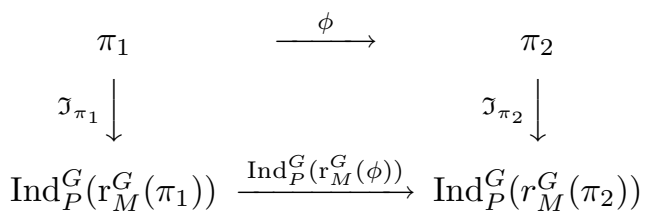

commutes.

In this paper we shall need only the first claim from the following lemma. Nevertheless, we also present the proof of the second claim of the lemma, since it is naturaly related to the problem considered in the lemma. 
8.2. Lemma. Let $\tau$ be an irreducible cuspidal representation of $M=M_{\Theta}$. Assume that $(M, \tau) \in \Omega(G)$ is regular and irreducible. Suppose that we have an exact sequence

$$
0 \rightarrow \operatorname{Ind}_{P}^{G}(\tau) \stackrel{f}{\rightarrow} \pi \stackrel{g}{\rightarrow} \operatorname{Ind}_{P}^{G}(\tau) \rightarrow 0
$$

of $G$-modules, such that $\pi$ is indecomposable. Then:

(i) The representation $\mathrm{r}_{M G}(\pi)$ is not semi-simple.

(ii) There exist $w \in W^{\Theta}$ and an indecomposable representation $\sigma$ of $M$ satisfying the exact sequence

$$
0 \rightarrow w \tau \rightarrow \sigma \rightarrow w \tau \rightarrow 0
$$

such that

$$
\pi \cong \operatorname{Ind}_{P}^{G}(\sigma)
$$

Proof. Denote

$$
\gamma=\operatorname{Ind}_{P}^{G}(\tau), \quad f^{\prime}=\operatorname{Ind}_{P}^{G}\left(r_{M}^{G}(f)\right), \quad g^{\prime}=\operatorname{Ind}_{P}^{G}\left(r_{M}^{G}(g)\right) .
$$

Now (8-1) and (8-2) imply that the following diagram is commutative:

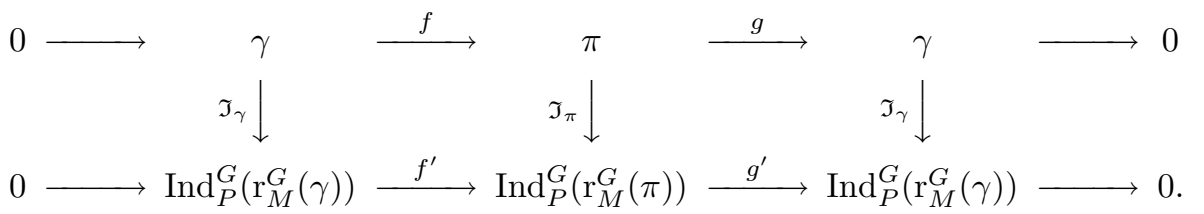

Moreover, we know that the rows are exact sequences.

Frobenius reciprocity $\operatorname{Hom}_{M}\left(\mathrm{r}_{M}^{G}(\gamma), \tau\right) \cong \operatorname{Hom}_{G}(\gamma, \gamma)$ and the fact that $\gamma \neq\{0\}$ imply $\mathrm{r}_{M}^{G}(\gamma) \neq\{0\}$. Thus, $\mathfrak{I}_{\gamma}$ is injective since $\gamma$ is irreducible. In the standard way we get that $\mathfrak{I}_{\pi}$ is injective $\left(\mathfrak{I}_{\pi}(v)=0 \Rightarrow g^{\prime} \mathfrak{I}_{\pi}(v)=0=\mathfrak{I}_{\gamma} g(v) \Rightarrow g(v)=0 \Rightarrow \exists u\right.$ such that $v=f(u) \Rightarrow 0=\mathfrak{I}_{\pi}(v)=\mathfrak{I}_{\pi} f(u) \Rightarrow f^{\prime} \mathfrak{I}_{\gamma}=0 \Rightarrow \mathfrak{I}_{\gamma}(u)=0 \Rightarrow u=0 \Rightarrow$ $v=f(u)=0)$. Thus,

$$
\pi \hookrightarrow \operatorname{Ind}_{P}^{G}\left(\mathrm{r}_{M}^{G}(\pi)\right)
$$

This implies that that $\mathrm{r}_{M}^{G}(\pi)$ is not semi-simple (otherwise, $\operatorname{Ind}_{P}^{G}\left(\mathrm{r}_{M}^{G}(\pi)\right.$ ) would be semi-simple, and then $\pi$ would be semi-simple). This completes the proof of (i).

(ii) We shall denote by s.s. $(\tau)$ the semi-simplification of an admissible representation $\tau$ of finite length. We shall view it here as the direct sum of all its irreducible subquotients (counted with multiplicities).

Since s.s. $(\pi)=2$ s.s. $(\gamma)$ by $(8-2)$, s.s. $\left(\mathrm{r}_{M}^{G}(\pi)\right)=2$ s.s. $\left(\mathrm{r}_{M}^{G}(\gamma)\right)$. Now using the Geometric Lemma of [BZ] or Theorem 6.3.5 of [C] we get

$$
\text { s.s. }\left(\mathrm{r}_{M}^{G}(\pi)\right)=2 \bigoplus_{w \in W^{\ominus}} w \tau \text {. }
$$

Because of regularity of $\tau$, all the $w \sigma$ 's have different infinitesimal characters (these are representations of $M$ ). Therefore,

$$
\mathrm{r}_{M}^{G}(\pi)=\bigoplus_{w \in W^{\ominus}} \sigma_{w}
$$

where $\sigma_{w}$ are representations of length two whose Jordan-Hölder series contain only $w \tau$. This implies that

$$
\pi \hookrightarrow \operatorname{Ind}_{P}^{G}\left(\bigoplus_{w \in W^{\ominus}} \sigma_{w}\right) \cong \bigoplus_{w \in W^{\ominus}} \operatorname{Ind}_{P}^{G}\left(\sigma_{w}\right) .
$$


Note that s.s. $\left(\operatorname{Ind}_{P}^{G}\left(\sigma_{w}\right)\right)=2 \gamma$. Denote by $p_{w_{0}}$ the projection of

$$
p_{w_{0}}: \bigoplus_{w \in W^{\ominus}} \operatorname{Ind}_{P}^{G}\left(\sigma_{w}\right) \rightarrow \operatorname{Ind}_{P}^{G}\left(\sigma_{w_{0}}\right) .
$$

Choose $w_{0} \in W^{\Theta}$ such that $p_{w_{0}} \mid \pi \neq 0$. Suppose that $p_{w_{0}} \mid \pi$ is not injective. Now (8$3)$ implies that there exists $w_{1} \in W^{\Theta}$ such that $p_{w_{1}} \mid \operatorname{Ker}\left(p_{w_{0}} \mid \pi\right) \neq 0$. If $p_{w_{1}} \mid \pi$ is not injective, then $\pi$ is a direct sum of $\operatorname{Ker}\left(p_{w_{0}} \mid \pi\right)$ and $\operatorname{Ker}\left(p_{w_{1}} \mid \pi\right)$, which contradicts the assumption of the indecomposability of $\pi$. Thus, $p_{w_{1}} \mid \pi$ is injective. Since the lengths of $\pi$ and $\operatorname{Ind}_{P}^{G}\left(\sigma_{w_{1}}\right)$ are both two, $p_{w_{1}} \mid \pi$ is also surjective, and therefore it is an isomorphism. Thus, $\pi \cong \operatorname{Ind}_{P}^{G}\left(\sigma_{w_{1}}\right)$. If $\sigma_{w_{1}}$ is decomposable, then $\operatorname{Ind}_{P}^{G}\left(\sigma_{w_{1}}\right)$ is decomposable, which contradicts the assumption of the indecomposability of $\pi$. Thus, $\sigma_{w}$ is indecomposable. This completes the proof of the lemma.

Denote

$$
\mathbb{L}=M / M^{0} .
$$

Recall that $\mathbb{L}$ is a free $\mathbb{Z}$-module of finite rank. Let

$$
p: M \rightarrow \mathbb{L}
$$

be the quotient mapping. Denote by

$$
p_{Z(M)}: Z(M) \rightarrow \mathbb{L}
$$

the restriction of $p$ to the center $Z(M)$ of $M$. Then $p_{Z(M)}$ has a finite cokernel and compact kernel (which is $Z(M) \cap M^{0}$ ).

Denote

$$
\mathbb{L}_{1}=Z(M) /\left(Z(M) \cap M^{0}\right)
$$

Observe that $Z(M) \hookrightarrow M$ induces a natural embedding

$$
\mathbb{L}_{1} \hookrightarrow \mathbb{L}
$$

We shall identify $\mathbb{L}_{1}$ with a sublattice of $\mathbb{L}$ by the above embedding. These two lattices have the same rank (which implies that $\mathbb{L} / \mathbb{L}_{1}$ is finite).

Observe that

$$
Z(M)=\left(Z(M) \cap M^{0}\right) \mathcal{L}_{1} .
$$

Using the fact that $Z(M) \cap M^{0}$ is compact, one gets that $\mathcal{L}_{1}$ is cocompact in $Z(M)$.

Observe that for $m \in M, m M^{0} m^{-1}=M^{0}$. This implies that $w M w^{-1}$ is well defined for $w \in W(\Theta)$, which implies $w M^{0} w^{-1}=M^{0}$. Thus, we have the action of $W(\Theta)$ on $\mathbb{L}=M / M^{0}$ given by the conjugation modulo $M^{0}$. Since the commutator subgroup of $M$ is contained in $M^{0}, W_{\Theta}$ acts trivially on $\mathbb{L}$. Therefore, $W^{\Theta}$ acts on $\mathbb{L}$.

For $w \in W(\Theta)$ and $z \in Z(M)$ we shall denote

$$
w \cdot z=w z w^{-1} .
$$

Clearly, this is well defined and defines an action of $W(\Theta)$ on $Z(M)$. Clearly, $W_{\Theta}$ acts trivially on $Z(M)$. Therefore $W^{\Theta}=W(\Theta) / W_{\Theta}$ acts on $Z(M)$. Since $Z(M) \cap M^{0}$ is obviously the maximal compact subgroup of $Z(M), W^{\Theta}$ preserves $Z(M) \cap M^{0}$. Therefore, we have the quotient action of $W^{\Theta}$ on $\mathbb{L}_{1}$.

By the above definitions, $p$ and $p_{Z(M)}$ commute with the action of $W^{\Theta}$. 
8.3. Lemma. There exists a $W^{\Theta}$-invariant (for conjugation) subgroup $\mathcal{L}_{0}$ of $Z(M)$ such that

is injective, and that

$$
p_{Z(M)} \mid \mathcal{L}_{0}
$$

has finite index in $\mathbb{L}_{1}$ (and thus also in $\mathbb{L}$ ).

$$
\mathbb{L}_{0}:=p_{Z(M)}\left(\mathcal{L}_{0}\right)
$$

Proof. Fix some splitting homomorphism of $p_{Z(M)}: Z(M) \rightarrow \mathbb{L}_{1}$, and let the image of $\mathbb{L}_{1}$ under the splitting homomorphism be

$$
\mathcal{L}_{1}
$$

Observe that $\mathcal{L}_{1} \subseteq Z(M), \mathcal{L}_{1} \cong \mathbb{L}_{1}$ and $p_{Z(M)} \mid \mathcal{L}_{1}$ is injective.

Denote by

$$
\mathcal{L}_{2}
$$

the subgroup of $Z(M)$ generated by $\bigcup_{w \in W^{\ominus}} w \cdot \mathcal{L}_{1}$. Then $\mathcal{L}_{2}$ is a finitely generating abelian group containing $\mathcal{L}_{1}$. It is $W^{\Theta}$-invariant by construction. We can take a positive integer $k$ such that

$$
\mathcal{L}_{3}:=\mathcal{L}_{2}^{k}=\left\{l^{k} ; k \in \mathcal{L}_{2}\right\}
$$

is without torsion. Obviously this subgroup is $W^{\Theta}$-invariant. Note that $\mathcal{L}_{3}$ is a free abelian group containing $\mathcal{L}_{1}^{k}$. This implies that $p_{Z(M)}\left(\mathcal{L}_{3}\right)$ has finite index in $\mathbb{L}_{1}=p_{Z(M)}\left(\mathcal{L}_{1}\right)$.

Fix a basis of the $\mathbb{Z}$-module $\mathcal{L}_{3}$. One gets in a standard way a positive definite symmetric $\mathbb{Z}$-bilinear form on $\mathcal{L}_{3}$ which takes values in $\mathbb{Z}$. Acting by $W^{\Theta}$ on such a form and then taking the sum of all such forms, one again gets a positive definite symmetric $\mathbb{Z}$-bilinear form which takes values in $\mathbb{Z}$, and which is invariant under the action of $W^{\Theta}$.

Fix such a $W^{\Theta}$-invariant positive definite symmetric $\mathbb{Z}$-bilinear form on $\mathcal{L}_{3}$ taking values in $\mathbb{Z}$. Let

$$
\mathcal{L}_{0}
$$

be the orthogonal complement in $\mathcal{L}_{3}$ of $\operatorname{Ker}\left(p_{z(M)} \mid \mathcal{L}_{3}\right)$. Then $\mathcal{L}_{0}$ is $W^{\Theta}$-invariant,

$$
\operatorname{Ker}\left(p_{Z(M)} \mid \mathcal{L}_{3}\right) \cap \mathcal{L}_{0}=0
$$

and

$$
\operatorname{Ker}\left(p_{Z(M)} \mid \mathcal{L}_{3}\right) \oplus \mathcal{L}_{0}
$$

has a finite index in $\mathcal{L}_{3}$. Therefore $p_{Z(M)}\left(\mathcal{L}_{0}\right)$ has a finite index in $p_{Z(M)}\left(\mathcal{L}_{3}\right)$ and then also in $\mathbb{L}_{1}$.

Denote

$$
\mathbb{L}_{0}=p_{Z(M)}\left(\mathcal{L}_{0}\right)
$$

Note that $p_{z(M)}$ induces an isomorphism of $\mathcal{L}_{0}$ onto $\mathbb{L}_{0}, \mathbb{L}_{0}$ is $W^{\Theta}$-invariant and $\mathbb{L}_{0}$ has a finite index in $\mathbb{L}_{1}$.

As we have already observed, we have a positive definite $W^{\Theta}$-invariant $\mathbb{Z}$-bilinear form on $\mathbb{L}_{0}$, which takes values in $\mathbb{Z}$. We shall fix such a form.

Take $a_{1}^{\prime} \in \mathbb{L}_{0} \backslash\{1\}$ and denote $X_{1}^{\prime}=W^{\Theta} . a_{1}^{\prime}$. Denote by $\mathbb{L}_{1}^{\prime}$ the subgroup of $\mathbb{L}_{0}$ generated by $X_{1}^{\prime}$. Let $\left(\mathbb{L}_{1}^{\prime}\right)^{\perp}$ be the orthogonal complement of $\mathbb{L}_{1}^{\prime}$ in $\mathbb{L}_{0}$ with respect to the fixed $W^{\Theta}$-invariant form. Then we know that $\left(\mathbb{L}_{1}^{\prime}\right)^{\perp}$ is $W^{\Theta}$-invariant and that $\mathbb{L}_{1}^{\prime} \oplus\left(\mathbb{L}_{1}^{\prime}\right)^{\perp}$ has a finite index in $\mathbb{L}_{0}$. Now choose $a_{2}^{\prime} \in\left(\mathbb{L}_{1}^{\prime}\right)^{\perp} \backslash\{1\}$, set $X_{2}^{\prime}=W^{\Theta} . a_{2}^{\prime}$ and denote by $\mathbb{L}_{2}^{\prime}$ the subgroup generated by $X_{2}^{\prime}$. Consider the 
orthogonal complement of $\mathbb{L}_{2}^{\prime}$ in $\left(\mathbb{L}_{1}^{\prime}\right)^{\perp}$. This process must end in a finite number of steps. In this way we shall get subgroups

$$
\mathbb{L}_{1}^{\prime}, \ldots, \mathbb{L}_{k}^{\prime}
$$

of $\mathbb{L}_{0}$ and subsets

$$
X_{1}^{\prime}, \ldots, X_{k}^{\prime}
$$

such that:

(1) The subgroup $\mathbb{L}^{\prime}$ generated by $\bigcup_{i=1}^{k} \mathbb{L}_{i}^{\prime}$ holds

$$
\mathbb{L}^{\prime}=\mathbb{L}_{1}^{\prime} \oplus \cdots \oplus \mathbb{L}_{k}^{\prime} .
$$

(2) $\mathbb{L}^{\prime}$ has a finite index in $\mathbb{L}_{1}$.

(3) Each $X_{i}^{\prime}$ is a $W^{\Theta}$-orbit and generates $\mathbb{L}_{i}^{\prime}$ as a group.

Denote

$$
\begin{aligned}
\mathcal{L}^{\prime} & =\left(p_{Z(M)} \mid \mathcal{L}_{0}\right)^{-1}\left(\mathbb{L}^{\prime}\right), \\
\mathcal{L}_{i}^{\prime} & =\left(p_{Z(M)} \mid \mathcal{L}_{0}\right)^{-1}\left(\mathbb{L}_{i}^{\prime}\right), \\
a_{i} & =\left(p_{Z(M)} \mid \mathcal{L}_{0}\right)^{-1}\left(a_{i}^{\prime}\right) .
\end{aligned}
$$

Thus, $\mathcal{L}^{\prime} \subseteq Z(M)$ is a free abelian group which is invariant for the action of $W^{\Theta}$. Further

$$
p_{Z(M)} \mid \mathcal{L}^{\prime}: \mathcal{L}^{\prime} \rightarrow \mathbb{L}^{\prime} \subseteq \mathbb{L}
$$

is an isomorphism which commutes with the action of $W^{\Theta}$, and $p_{Z(M)}\left(\mathcal{L}^{\prime}\right)=\mathbb{L}^{\prime}$ has finite index in $\mathbb{L}$.

Let $x, y \in \mathbb{L}, x \neq y$ and $c \in \mathbb{C}$. Then one directly sees that there exists $\chi \in \Psi(M)$ such that

$$
\chi(x) \neq c \chi(y)
$$

(the case $c \neq 1$ is evident, for the other case use the fact that the transcendence degree of $\mathbb{C}$ over $\mathbb{Q}$ is infinite). Moreover, the set of all such $\chi$ is a Zariski open subset of $\Psi(M)$ (recall that $\Psi(M)$ is an irreducible algebraic variety).

Let $\rho$ be an irreducible cuspidal representation of $M$. Denote

$$
\Psi(M)_{\rho, \operatorname{diff}}=\left\{\chi \in \Psi(M) ; \text { if } 1 \leq i \leq k \text { and } w_{1}, w_{2} \in W^{\Theta} \text { such that } w_{1} . a_{i} \neq w_{2} . a_{i},\right.
$$

$$
\text { then } \left.c_{\rho}\left(w_{1} . a_{i}\right) \chi\left(w_{1} . a_{i}\right) \neq c_{\rho}\left(w_{2} . a_{i}\right) \chi\left(w_{2} . a_{i}\right)\right\} \text {. }
$$

Since $w_{1} \cdot a_{i} \neq w_{2} . a_{i}$ implies $p\left(w_{1} \cdot a_{i}\right) \neq p\left(w_{2} . a_{i}\right)$, which implies $w_{1} \cdot a_{i}^{\prime} \neq w_{2} \cdot a_{i}^{\prime}$,

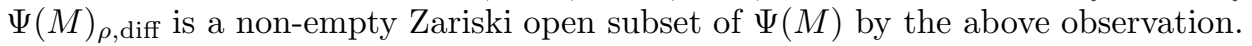

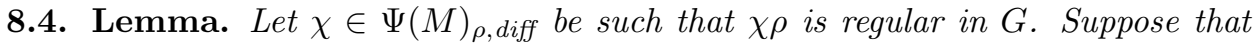
we have an exact sequence

$$
0 \rightarrow \chi \rho \stackrel{\alpha}{\rightarrow} \sigma \stackrel{\beta}{\rightarrow} \chi \rho \rightarrow 0
$$

of $M$-modules. If $\operatorname{Ind}_{P}^{G}(\sigma)$ has an infinitesimal character, then the above sequence splits.

Proof. We shall suppose that $\operatorname{Ind}_{P}^{G}(\sigma)$ has an infinitesimal character, but that the above sequence does not split. We shall see in a sequence of steps that this assumption leads to a contradiction.

(1) First observe that (v) of Remarks 3.10 implies that $\sigma$ does not have a central character. 
(2) Now we shall prove that $\mathcal{L}^{\prime}$ does not act by scalar operators. Suppose that $\mathcal{L}^{\prime}$ acts by scalars (in $\sigma$ ). Using the fact that $Z(M) / \mathcal{L}^{\prime}$ is compact (which follows from the compactness of $Z(M) / \mathcal{L}_{1}$ ), one easily sees that there is a basis of the representation space of $\sigma$ in which $Z(M)$ acts diagonally. This implies that $Z(M)$ acts on each of these one-dimensional space as the central character $c_{\chi \rho}$ of $\chi \rho$. Thus, $Z(M)$ acts by scalar operators. This cannot happen by (1).

(3) Let $\mathfrak{z} \in \mathfrak{Z}(G)$. By Proposition $3.8, \mathfrak{z}$ acts in $\operatorname{Ind}_{P}^{G}(\sigma)$ as $\operatorname{Ind}_{P}^{G}\left(\sigma\left(\mathrm{i}_{G M}^{*}(\mathfrak{z})\right)\right)$. Note that $\operatorname{Ind}_{P}^{G}\left(\sigma\left(\mathrm{i}_{G M}^{*}(\mathfrak{z})\right)\right)(f)=\sigma\left(\mathrm{i}_{G M}^{*}(\mathfrak{z})\right) \circ f$ for $f \in \operatorname{Ind}_{P}^{G}(\sigma)$. Since $f \mapsto f(1)$ is an epimorphism of $\operatorname{Ind}_{P}^{G}(\sigma)$ onto $\sigma$ and $\mathfrak{z}$ acts as a scalar operator in $\operatorname{Ind}_{P}^{G}(\sigma)$, we directly get that $\mathrm{i}_{G M}^{*}(\mathfrak{z})$ act as a scalar operator in $\sigma$. Obviously, the scalar by which we multiply both representations is the same.

(4) Take any $z \in Z(M)$. As we have seen in (ii) (and (iii)) of Remarks 3.10, z defines in a natural way $z_{M} \in \mathcal{Z}(M)$, which corresponds to

$$
\left(z_{M}\right)^{\wedge}: \Omega(M) \rightarrow \mathbb{C}, \quad\left(M^{\prime}, \tau\right) \mapsto c_{\tau}(z),
$$

of $\mathfrak{Z}(M)$. Let $z_{M}^{\prime}$ be an element of $\mathfrak{Z}(M)$ such that $\left(z_{M}^{\prime}\right)^{\wedge}$ coincides with $\left(z_{M}\right)^{\wedge}$ on cuspidal components of $\Omega(M)$, and that it is zero on non-cuspidal components.

Denote the representation spaces of $\chi \rho$ and $\sigma$ by $U$ and $U^{\prime}$, respectively. Then

$$
(\chi \rho)\left(z_{M}^{\prime}\right)=(\chi \rho)\left(z_{M}\right)=(\chi \rho)(z)=c_{\chi \rho}(z) \operatorname{id}_{U} \quad \text { and } \quad \sigma\left(z_{M}^{\prime}\right)=\sigma\left(z_{M}\right)=\sigma(z) .
$$

(This follows from the fact that if a smooth representation $\pi$ of $M$ is supported by some connected component $\Omega \subseteq \Omega(M)$, then the action of $\pi(\mathfrak{z}), \mathfrak{z} \in \mathfrak{Z}(G)$, depends only on the restriction $\mathfrak{z} \mid X$; for this simple observation see the proof of Proposition 4.6 in $\mathrm{T}$. .)

Clearly, the action of $W^{\Theta}$ on $M$ (by conjugation) sends $Z(M)$ to itself. For $z \in Z(M)$ denote it by

$$
z_{W^{\Theta}}^{\prime}=\sum_{w \in W^{\Theta}}(w \cdot z)_{M}^{\prime} .
$$

Then

$$
\left(z_{W^{\Theta}}^{\prime}\right)^{\wedge}: \Omega(M) \rightarrow \mathbb{C}, \quad(M, \tau) \mapsto \sum_{w \in W^{\Theta}} c_{\tau}(w . z)
$$

and on non-cuspidal components it is zero.

Look at the natural mapping $\mathrm{i}_{G M}: \Omega(M) \rightarrow \Omega(G)$ introduced in the third section. Suppose that $\mathrm{i}_{G M}\left(\left(M^{\prime}, \tau^{\prime}\right)\right)=\mathrm{i}_{G M}\left(\left(M^{\prime \prime}, \tau^{\prime \prime}\right)\right)$. This implies that $\left(M^{\prime}, \tau^{\prime}\right)$ and $\left(M^{\prime \prime}, \tau^{\prime \prime}\right)$ are conjugate in $G$. If $M^{\prime}$ is a proper Levi subgroup of $M$, then obviously $M^{\prime \prime}$ is also a proper Levi subgroup of $M$, and we therefore have

$$
\left(z_{W^{\Theta}}^{\prime}\right)^{\wedge}\left(\left(M^{\prime}, \tau^{\prime}\right)\right)=\left(z_{W^{\Theta}}^{\prime}\right)^{\wedge}\left(\left(M^{\prime \prime}, \tau^{\prime \prime}\right)\right)=0
$$

by the definition of $z_{W^{\Theta}}^{\prime}\left(\right.$ and $\left.\left(z_{W^{\Theta}}^{\prime}\right)^{\wedge}\right)$. Suppose $M^{\prime}=M$. Then $M^{\prime \prime}=M$. Further, $\tau^{\prime}=w_{0} \tau^{\prime \prime}$ for some $w_{0} \in W^{\Theta}$. Now one easily shows that

$$
\begin{aligned}
& \left(z_{W^{\Theta}}^{\prime}\right)^{\wedge}\left(\left(M, \tau^{\prime}\right)\right)=\sum_{w \in W^{\Theta}} c_{\tau^{\prime}}(w \cdot z)=\sum_{w \in W^{\Theta}} c_{w \tau^{\prime}}(z) \\
& =\sum_{w \in W^{\Theta}} c_{w w_{0} \tau^{\prime \prime}}(z)=\sum_{w \in W^{\Theta}} c_{w \tau^{\prime \prime}}(z)=\sum_{w \in W^{\Theta}} c_{\tau^{\prime \prime}}(w . z)=\left(z_{W^{\Theta}}^{\prime}\right)^{\wedge}\left(\left(M, \tau^{\prime \prime}\right)\right) .
\end{aligned}
$$

This implies that there exists a regular function $\mathfrak{z}_{z}^{\prime}$ on $\Omega(G)$ (i.e. $\mathfrak{z} z \in \mathfrak{Z}(G)$ ) such that $z_{W^{\Theta}}^{\prime}$ factors through $\mathrm{i}_{G M}: \Omega(M) \rightarrow \Omega(G)$, i.e. that

$$
z_{W}^{\prime}=\mathfrak{z}_{z}^{\prime} \circ \mathrm{i}_{G M} \text {. }
$$


Thus

$$
\mathrm{i}_{G M}^{*}\left(\mathfrak{z}_{z}^{\prime}\right)=z_{W^{\Theta}}^{\prime} .
$$

We have $\left(\mathfrak{z}_{z}^{\prime}\right)^{\wedge}: \Omega(G) \rightarrow \mathbb{C}$ and obviously

$$
\left(\mathfrak{z}_{z}^{\prime}\right)^{\wedge}((M, \tau))=\sum_{w \in W^{\ominus}} c_{\tau}(w \cdot z) .
$$

Observe that

$$
\sigma\left(\hat{z}_{W^{\ominus}}^{\prime}\right)=\sum_{w \in W^{\ominus}} \sigma(w \cdot z) .
$$

Since $z_{W^{\ominus}}^{\prime}=\mathrm{i}_{G M}^{*}\left(\mathfrak{z}_{z}^{\prime}\right),(3)$ and the exact sequence in the lemma imply

$$
\sigma\left(\hat{z}_{W^{\ominus}}^{\prime}\right)=\sigma\left(\mathrm{i}_{G M}^{*}\left(\mathfrak{z}_{z}^{\prime}\right)\right)=\left(\sum_{w \in W^{\ominus}} c_{\chi \rho}(w \cdot z)\right) \operatorname{id}_{U^{\prime}} .
$$

Thus

$$
\sigma\left(\hat{z}_{W^{\Theta}}^{\prime}\right)=\sum_{w \in W^{\Theta}} \sigma(w . z)=\left(\sum_{w \in W^{\ominus}} \chi(w \cdot z) c_{\rho}(w \cdot z)\right) \operatorname{id}_{U^{\prime}} .
$$

(5) We have denoted by $U$ and $U^{\prime}$ the representation spaces of $\chi \rho$ and $\sigma$, respectively. Let $\beta^{\prime}$ be a splitting homomorphism of $\beta$, considered as a linear map only (i.e. $\beta^{\prime}$ does not need to be an $M$-intertwining, and it is not, since we suppose that $\sigma$ is indecomposable). After identifications, we can consider $\alpha$ and $\beta^{\prime}$ as inclusions. Therefore,

$$
U^{\prime}=U \oplus U
$$

(the first summand is $M$-invariant, while the second one is not). Denote

$$
e_{1}=\alpha, \quad e_{2}=\beta^{\prime},
$$

and the projection of $U^{\prime}$ on the $i$-th summand by

$$
q_{i} .
$$

Clearly

$$
q_{2}=\beta
$$

Observe

$$
\begin{gathered}
e_{1} q_{1}+e_{2} q_{2}=\operatorname{id}_{U^{\prime}}, \\
q_{i} e_{i}=\operatorname{id}_{U}, \quad q_{i} e_{3-1}=0, \quad i=1,2 .
\end{gathered}
$$

For a linear operator $A$ on $U^{\prime}$ denote $A_{i j}=q_{i} A e_{j}$ and $A^{\#}=\left[A_{i j}\right]_{1 \leq i, j \leq 2}$. Then for two operators we have $\left(A_{1} A_{2}\right)^{\#}=A_{1}^{\#} A_{2}^{\#}$.

Since $\alpha$ is intertwining, we have

$$
\sigma(m)_{11}=q_{1} \sigma(m) e_{1}=q_{1} \sigma(m) \alpha=q_{1} \alpha \chi \rho(m)=q_{1} e_{1} \chi \rho(m)=\chi \rho(m)
$$

and

$$
\sigma(m)_{21}=q_{2} \sigma(m) e_{1}=q_{2} \sigma(m) \alpha=q_{2} \alpha \chi \rho(m)=q_{2} e_{1} \chi \rho(m)=0 .
$$

Further,

$$
\sigma(m)_{22}=q_{2} \sigma(m) e_{2}=\beta \sigma(m) e_{2}=\chi \rho(m) \beta e_{2}=\chi \rho(m) q_{2} e_{2}=\chi \rho(m) .
$$


Thus

$$
\sigma(m)^{\#}=\left[\begin{array}{cc}
\chi \rho(m) & 0 \\
0 & \chi \rho(m)
\end{array}\right]\left[\begin{array}{cc}
\operatorname{id}_{U} & \Lambda(m) \\
0 & \operatorname{id}_{U}
\end{array}\right]=\left[\begin{array}{cc}
\chi \rho(m) & \chi \rho(m) \Lambda(m) \\
0 & \chi \rho(m)
\end{array}\right]
$$

for some linear operator $\Lambda(m)$ on $U$.

From $\sigma\left(z_{1}\right)^{\#} \sigma\left(z_{2}\right)^{\#}=\sigma\left(z_{1} z_{2}\right)^{\#}$ one gets

$$
\Lambda\left(z_{1} z_{2}\right)=\Lambda\left(z_{1}\right)+\Lambda\left(z_{2}\right)
$$

for $z_{1}, z_{2} \in Z(M)$ (recall that the elements of $Z(M)$ act by scalar operators in $U)$. Further, for $z \in Z(M)$ and $m \in M, \sigma(z)^{\#} \sigma(m)^{\#}=\sigma(m)^{\#} \sigma(z)^{\#}$. This and the Schur lemma imply that $\Lambda(z)$ is a scalar operator for $z \in Z(M)$. Thus, there exists a function $\lambda$ on $Z(M)$ such that $\Lambda(z)=\lambda(z) \operatorname{id}_{U}$. The above relation $\Lambda\left(z_{1} z_{2}\right)=\Lambda\left(z_{1}\right)+\Lambda\left(z_{2}\right)$ implies that $\lambda: Z \rightarrow \mathbb{C}$ is a homomorphism.

(6) Denote

$$
\lambda_{\mathcal{L}^{\prime}}=\lambda \mid \mathcal{L}^{\prime}
$$

Note that $\lambda_{\mathcal{L}^{\prime}}=0$ implies that $\mathcal{L}^{\prime}$ acts by scalars in $\sigma$, which can happen by (2). Thus, $\lambda_{\mathcal{L}^{\prime}} \neq 0$. Since $\mathcal{L}_{i}^{\prime}$ generates $\mathcal{L}^{\prime}, \lambda_{\mathcal{L}^{\prime}} \mid \mathcal{L}^{\prime}{ }_{i} \neq 0$ for some $i$. Since $X_{i}$ generates $\mathcal{L}^{\prime}{ }_{i}$ as a group, $\lambda_{\mathcal{L}^{\prime}} \mid X_{i} \neq 0$. Denote by

$$
W_{i}^{\Theta}
$$

the stabilizer of $a_{i}$ in $W^{\Theta}$.

For $z \in \mathcal{L}^{\prime}$, the relation $\sigma\left(\hat{z}_{W^{\Theta}}^{\prime}\right)=\sum_{w \in W^{\Theta}} \sigma(w . z)$ implies

$$
\sigma\left(\hat{z}_{W^{\ominus}}^{\prime}\right)=\sum_{w \in W^{\Theta}}\left[\begin{array}{cc}
c_{\chi \rho}(w . z) \operatorname{Id}_{U} & c_{\chi \rho}(w . z) \lambda_{\mathcal{L}^{\prime}}(w . z) \operatorname{id}_{U} \\
0 & c_{\chi \rho}(w . z) \operatorname{Id}_{U}
\end{array}\right] .
$$

Since $\sigma\left(\hat{z}_{W^{\Theta}}^{\prime}\right)$ is a scalar operator, we have

$$
\sum_{w \in W^{\Theta}} c_{\chi \rho}(w . z) \lambda_{\mathcal{L}^{\prime}}(w . z)=\sum_{w \in W^{\ominus}} \chi(w . z) c_{\rho}(w . z) \lambda_{\mathcal{L}^{\prime}}(w . z)=0
$$

(if this is not zero, then $\sigma\left(\hat{z}_{W^{\Theta}}^{\prime}\right)$ does not act as a multiplication by a scalar on each $\left.e_{2}(u), u \in U \backslash\{0\}\right)$. Therefore

$$
\sum_{w \in W^{\Theta}} \chi\left(w \cdot a_{i}^{k}\right) c_{\rho}\left(w \cdot a_{i}^{k}\right) \lambda_{\mathcal{L}^{\prime}}\left(w \cdot a_{i}^{k}\right)=0, \quad k=1,2, \ldots .
$$

Using the fact that $\lambda_{\mathcal{L}^{\prime}}$ is additive, we get

$$
\sum_{w \in W^{\ominus}}\left(\chi\left(w \cdot a_{i}\right) c_{\rho}\left(w \cdot a_{i}\right)\right)^{k} \lambda_{\mathcal{L}^{\prime}}\left(w \cdot a_{i}\right)=0, \quad k=1,2, \ldots
$$

and further

$$
\sum_{w \in W^{\ominus} / W_{i}^{\ominus}}\left(\chi\left(w \cdot a_{i}\right) c_{\rho}\left(w \cdot a_{i}\right)\right)^{k} \lambda_{\mathcal{L}^{\prime}}\left(w \cdot a_{i}\right)=0, \quad k=1,2, \ldots .
$$

Recall that for at least one $w \in W^{\Theta}$ we have

$$
\lambda_{\mathcal{L}^{\prime}}\left(w \cdot a_{i}\right) \neq 0 .
$$

If we consider the homogeneous system

$$
\sum_{w \in W^{\Theta} / W^{\Theta}}\left(\chi\left(w \cdot a_{i}\right) c_{\rho}\left(w \cdot a_{i}\right)\right)^{k} x_{i}=0, k=1,2, \ldots, \operatorname{card}\left(W^{\Theta} / W^{\Theta}{ }_{i}\right),
$$


then one easily sees that the determinant of it is non-zero. Namely, the determinant of the system is a non-zero multiple of a van der Monde determinant. This van der Monde determinant factors as a product of factors

$$
\left(\chi\left(w^{\prime} \cdot a_{i}\right) c_{\rho}\left(w^{\prime} \cdot a_{i}\right)-\chi\left(w^{\prime \prime} \cdot a_{i}\right) c_{\rho}\left(w^{\prime \prime} \cdot a_{i}\right)\right),
$$

with different $w^{\prime}, w^{\prime \prime} \in W^{\Theta} / W_{i}^{\Theta}$. Then since $w^{\prime} a_{i}$ and $w^{\prime \prime} a_{i}$ are different, the

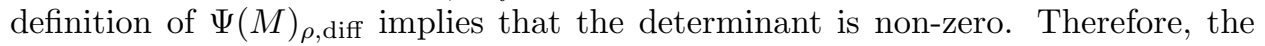
above system does not have a non-zero solution. This contradicts (8-6) and (8-7).

The proof of the lemma is now complete.

Denote by $\Psi(M)_{\rho, \text { reg }}\left(\right.$ resp. $\Psi(M)_{\rho \text {,irr }}$ ) the set of all $\chi$ in $\Psi(M)$ such that $\chi \rho$ is regular in $G$ (resp. $\operatorname{Ind}_{P}^{G}(\chi \rho)$ is irreducible). Then these two sets are non-empty Zariski open subsets of $\Psi(M)$. Let

$$
\begin{gathered}
\Psi(M)_{\rho, \text { diff }}^{W^{\ominus}}=\bigcap_{w \in W^{\ominus}} w \cdot \Psi(M)_{\rho, \text { diff }}, \\
\Psi(M)_{\rho, \text { reg }}^{W^{\ominus}}=\bigcap_{w \in W^{\ominus}} w \cdot \Psi(M)_{\rho, \text { reg }}, \\
\Psi(M)_{\rho, \text { irr }}^{W^{\ominus}}=\bigcap_{w \in W^{\ominus}} w \cdot \Psi(M)_{\rho, \text { irr }}, \\
\Psi(M)_{\rho, \text { s.s. }}=\Psi(M)_{\rho, \text { diff }}^{W^{\ominus}} \Psi \Psi(M)_{\rho, \text { reg }}^{W^{\ominus}} \cap \Psi(M)_{\rho, \text { irr }}^{W^{\ominus}}
\end{gathered}
$$

Note that $\Psi(M)_{\rho \text {,s.s. }}$ is a Zariski open dense subset of $\Psi(M)$.

8.5. Theorem. Let $(M, \rho) \in \Omega(G)$ and let $\chi \in \Psi(M)_{\rho, s . s .}$. Suppose that $\pi$ is a smooth representation with infinitesimal character equal to the evaluation in $(M, \chi \rho)$. Then $\pi$ is a direct sum of irreducible representations, each of which is isomorphic to $\operatorname{Ind}_{P}^{G}(\chi \rho)$.

Proof. By Lemma 8.1 it is enough to prove semi-simplicity for $\pi$ of length 2 .

Suppose that we have have a length two admissible representation $\pi$ of $G$ with infinitesimal character equal to evaluation in $(M, \chi \rho)$ which is indecomposable, with $\chi \in \Psi(M)_{\rho \text {,s.s. }}$. Then by (i) of Lemma 8.2 there exist $w \in W^{\Theta}$ and an indecomposable representation $\sigma$ of $M$ of length two satisfying the exact sequence

$$
0 \rightarrow w(\chi \rho) \rightarrow \sigma \rightarrow w(\chi \rho) \rightarrow 0 .
$$

This cannot happen by the previous lemma, and by the definition of $\Psi(M)_{\rho, \text { s.s. }}$. Thus, $\pi$ is decomposable.

By the definition of $\Psi(M)_{\rho, \text { s.s. }}$, each irreducible subquotient must be isomorphic to $\operatorname{Ind}_{P}^{G}(\chi \rho)$.

Since the quotient mapping $\chi \mapsto \chi \rho$ from $\Psi(M)$ onto the connected component of $\Omega(G)$ is open, the above theorem implies Theorem 3.9.

\section{ACKNOWLEDGMENTS}

The first author thanks Nolan Wallach for stimulating discussions, and the second author thanks Dragan Miličić and Peter Schneider for useful observations related to the problems studied in this paper. Part of this work was done while the second author enjoying the hospitality of the Hong Kong University of Science and Technology. 
At the end, we are very thankful to the referee for a number of very useful corrections and suggestions. These suggestions helped to make the paper much more readable.

\section{REFERENCES}

[BD] Bernstein, J. and Deligne, P., Le "center" de Bernstein, Représentations des groupes reductifs sur un corps local (1985), 1-32, Hermann, Paris. MR86e:22028

[BDK] Bernstein, J., Deligne, P. and Kazhdan, D., Trace Paley-Wiener theorem for reductive p-adic groups, Journal D'analyse Mathématique 47 (1986), 180-192. MF 88g:22016

[BR] Bernstein, J. and Rumelhart, K. Representations of p-adic groups, Lectures by Joseph Bernstein, preprint.

[BZ] Bernstein, I. N. and Zelevinsky, A.V., Induced representations of reductive p-adic groups I, Ann. Sci. École Norm Sup. 10 (1977), 441-472. MR58:28310

[C] Casselman, W. Introduction to the theory of admissible representations of p-adic reductive groups, preprint.

[HC1] Harish-Chandra (notes by G. van Dijk), Harmonic Analysis on Reductive p-adic Groups, Lecture Notes in Math. 162, Springer-Verlag, Berlin, 1970. MR54:2889

[HC2] Harish-Chandra, Harmonic analysis on reductive p-adic groups, Proc. Sympos. Pure Math. XXVI, Amer. Math. Soc., Providence, 1973, 167-192. MR 49:5238

[HOW] Huang, J.-S., Oshima, T. and Wallach, N., Dimensions of spaces of generalized spherical functions, Amer. J. Math. 118 (1996), 637-652. MR.97d:22015

[K] Kutzko, P. (J. Tiran, D. Vogan and J. Wolf, eds.), Smooth representations of reductive $p$ adic groups: An introduction to the theory of types, Geometry and Representation Theory of Real and p-adic Groups, Birkhauser, Boston, 1997, 175-196. MR.98k:22072

[MT] Moy, A. and Tadić, M., The Bernstein center in terms of invariant locally integrable functions, Represent. Theory 6 (2002), 313-329.

[T] Tadić, M., Geometry of dual spaces of reductive groups (non-archimedean case), J. Analyse Math. 51 (1988), 139-181. MR90c:22057

[W] Waldspurger, J.-L., La formule de Plancherel pour les groupes p-adiques, d'après HarishChandra, Journal de l'Institut de Math. de Jussieu 2 2, (2003), 235-333.

Department of Mathematics, The Hong Kong University of Science and Technology, Clear Water Bay, Kowloon, Hong Kong

E-mail address: mahuang@uxmail.ust.hk

Department of Mathematics, University of Zagreb, Bijenička 30, 10000 Zagreb, CroaTIA

E-mail address: tadic@math.hr 